\title{
Zeros of derivatives of strictly non-real meromorphic functions
}

\author{
J.K. Langley
}

February 21, 2020

\begin{abstract}
A number of results are proved concerning the existence of non-real zeros of derivatives of strictly non-real meromorphic functions in the plane. MSC 2000: 30D35.
\end{abstract}

\section{Introduction}

Let $f$ be a meromorphic function in the plane and let $\widetilde{f}(z)=\overline{f(\bar{z})}$ (this notation will be used throughout). Here $f$ is called real if $\widetilde{f}=f$, and strictly non-real if $\widetilde{f}$ is not a constant multiple of $f$. There has been substantial research concerning non-real zeros of derivatives of real entire or real meromorphic functions [1, 2, 4, 13, 14, 19, 22, 27, 28], but somewhat less in the strictly non-real case. The following theorem was proved in [12].

Theorem 1.1 ([12]) Let $f$ be a strictly non-real meromorphic function in the plane with only real poles. Then $f, f^{\prime}$ and $f^{\prime \prime}$ have only real zeros if and only if $f$ has one of the following forms:

$$
\begin{aligned}
(I) \quad f(z) & =A e^{B z} ; \\
(I I) & f(z)=A\left(e^{i(c z+d)}-1\right) ; \\
(I I I) & f(z)=A \exp (\exp (i(c z+d))) ; \\
(I V) & f(z)=A \exp [K(i(c z+d)-\exp (i(c z+d)))] ; \\
(V) & f(z)=\frac{A \exp [-2 i(c z+d)-2 \exp (2 i(c z+d))]}{\sin ^{2}(c z+d)} ; \\
(V I) & f(z)=\frac{A}{e^{i(c z+d)}-1} .
\end{aligned}
$$

Here $A, B \in \mathbb{C}$, while $c, d$ and $K$ are real with $c B \neq 0$ and $K \leq-1 / 4$.

The first aim of the present paper is to prove a result in the spirit of Theorem 1.1, but with no assumption on the location of poles. In [15, 16, 17] Hinkkanen determined all meromorphic functions $f$ in the plane such that $f$ and all its derivatives have only real zeros, using the fact that under these hypotheses $f$ has at most two distinct poles, by the Pólya shire theorem [10, Theorem 3.6]. For strictly non-real functions, the following two theorems will be proved. 
Theorem 1.2 Let $f$ be a strictly non-real meromorphic function in the plane such that all but finitely many zeros of $f^{(m)}$ are real for $m=0, \ldots, 12$. Then either $f^{\prime} / f$ is a rational function or

$$
f(z)=B\left(1-T(z) e^{i A z}\right), \quad A \in \mathbb{R}, \quad B \in \mathbb{C}, \quad A B \neq 0,
$$

where $T$ is a rational function with $|T(x)|=1$ for all $x \in \mathbb{R}$.

If, in addition, $f, f^{\prime}, f^{\prime \prime}$ and $f^{\prime \prime \prime}$ have only real zeros, then $f$ is given by one of the following, in which $a, b, c, d \in \mathbb{C}$ and $\mu \in \mathbb{Z}$ :

$$
\text { (i) } \quad f(z)=(a z+b)^{\mu} ; \quad \text { (ii) } \quad f(z)=\frac{a z+b}{c z+d} ; \quad \text { (iii) } \quad f(z)=e^{a z+b}-c .
$$

Theorem 1.3 Let $f$ be a strictly non-real meromorphic function in the plane such that all zeros of $f^{(m)}$ are real for $m=0, \ldots, 9$. Then $f$ is given by (2).

It is very unlikely that Theorems 1.2 and 1.3 are sharp in terms of the number of derivatives considered, but examples (III)-(VI) of Theorem 1.1] show that the absence of non-real zeros of $f$, $f^{\prime}$ and $f^{\prime \prime}$ is not enough to imply (2).

The next result concerns the determination of all meromorphic functions $f$ in the plane such that $f$ and $f^{\prime \prime}$ have only real zeros and poles (thus discarding the hypothesis in Theorem 1.1 that $f^{\prime}$ has only real zeros). Such a classification is not known in the real meromorphic case, except when $f$ has finitely many poles [2, 28], or finitely many zeros [23], but for strictly nonreal functions of finite lower order the problem is solved by the following theorem, in which the terminology is from [10].

Theorem 1.4 Let $f$ be a strictly non-real meromorphic function in the plane, such that all but finitely many zeros and poles of $f$ and $f^{\prime \prime}$ are real. Then $f$ satisfies, as $r \rightarrow \infty$,

$$
\bar{N}(r, f)+\bar{N}(r, 1 / f)=O(r) \text { and } T\left(r, f^{\prime} / f\right)=O(r \log r) .
$$

If, in addition, $f$ has finite lower order and all zeros and poles of $f$ and $f^{\prime \prime}$ are real then $f$ is given by one of

$$
\begin{aligned}
\text { (a) } f(z) & =e^{A_{1} z+B_{1}}, \\
\text { (b) } f(z) & =e^{i a_{1} z}\left(T_{1}^{\prime}(z) \sin \left(a_{1} z+b_{1}\right)-T_{1}(z) \cos \left(a_{1} z+b_{1}\right)\right), \\
\text { (c) } f(z) & =\frac{T_{1}(z)}{e^{2 i\left(a_{1} z+b_{1}\right)}-1},
\end{aligned}
$$

in which $A_{1}, B_{1} \in \mathbb{C}$ and $a_{1}, b_{1} \in \mathbb{R}$, while $T_{1}$ is a polynomial of degree at most 1 such that $T_{1}(z)=0$ implies $\sin \left(a_{1} z+b_{1}\right)=0$.

If $T_{1}$ is a non-zero constant in (b) or (c) of (4) then $f$ reduces to (II) or (VI) of Theorem 1.1 and $f, f^{\prime}$ and $f^{\prime \prime}$ all have only real zeros and poles. However, $T_{1}$ is non-constant in both of the following examples:

$$
\begin{aligned}
& f_{1}(z)=e^{i z}(\sin z-z \cos z), \quad f_{1}^{\prime}(z)=e^{i z}(z \sin z+i(\sin z-z \cos z)), \quad f_{1}^{\prime \prime}(z)=2 z e^{2 i z} ; \\
& f_{2}(z)=\frac{z}{e^{2 i z}-1}, \quad f_{2}^{\prime}(z)=\frac{(1-2 i z) e^{2 i z}-1}{\left(e^{2 i z}-1\right)^{2}}, \quad f_{2}^{\prime \prime}(z)=\frac{(4 i-4 z) e^{2 i z}-(4 i+4 z) e^{4 i z}}{\left(e^{2 i z}-1\right)^{3}} .
\end{aligned}
$$


Here $f_{1}^{\prime}$ and $f_{2}^{\prime}$ each have infinitely many non-real zeros, but $f_{2}^{\prime \prime}(z)=0$ forces

$$
e^{2 i z}=\frac{4 i-4 z}{4 i+4 z}, \quad z=\tan z
$$

all solutions of which are real (see Lemma 2.7), as are all zeros of $f_{1}$. Furthermore, writing

$$
\frac{f_{3}^{\prime}(z)}{f_{3}(z)}=\frac{1}{i+z}+i e^{i z}, \quad \frac{f_{3}^{\prime \prime}(z)}{f_{3}(z)}=\left(\frac{i-z}{i+z}\right) e^{i z}-e^{2 i z}
$$

defines a strictly non-real entire function $f_{3}$ of infinite order, with one zero, for which $f_{3}^{\prime}$ has infinitely many non-real zeros, while all but finitely many zeros of $f_{3}^{\prime \prime}$ are real by [24, Lemma 2.3].

The author thanks John Rossi for helpful discussions, and the referee for a very careful reading of the manuscript and several helpful suggestions.

\section{Preliminaries}

The following theorem is a combination of results from [7, 8, 20] and uses notation from [10].

Theorem 2.1 ([7, 8, 20]) Let $h$ be a non-constant meromorphic function in the plane.

(i) For $n \geq 3$ there exists $c_{n}>0$, depending only on $n$, such that

$$
T\left(r, h^{\prime} / h\right) \leq c_{n}\left(\bar{N}(r, 1 / h)+\bar{N}\left(r, 1 / h^{(n)}\right)\right)+O(\log r) \quad \text { as } r \rightarrow \infty .
$$

(ii) If $n \geq 2$ and $h$ and $h^{(n)}$ have finitely many zeros, then $h^{\prime} / h$ is a rational function: equivalently, $h=S e^{Q}$ with $S$ a rational function and $Q$ a polynomial.

Here part (i) follows from [8, Theorem 3] (which should be stated for functions which have transcendental logarithmic derivative, rather than merely being themselves transcendental), and part (ii) was proved in [7, 20].

Theorem 2.2 ([3]) Let $k \geq 2$ and let $\mathcal{H}$ be a family of functions meromorphic on a plane domain $D$ such that $h h^{(k)}$ has no zeros in $D$, for each $h \in \mathcal{F}$. Then the family $\left\{h^{\prime} / h: h \in \mathcal{H}\right\}$ is normal on $D$.

Lemma 2.1 ([25]) Let $h$ be a transcendental meromorphic function in the plane such that $h^{\prime} / h$ has finite lower order and $h^{\prime} / h$ and $h^{\prime \prime} / h^{\prime}$ have finitely many zeros. Then $h^{\prime \prime} / h^{\prime}$ is a rational function and $h$ has finite order and finitely many poles.

The next two lemmas involve Tsuji's analogue [29] for the upper half-plane of Nevanlinna's characteristic function, which was developed further by Levin and Ostrovskii [26] (see also [2, 9]). The first is directly related to Theorem [2.1(i) and was deduced in [22] from Frank's method [7].

Lemma 2.2 ([22, 24]) Let $f$ be a non-constant meromorphic function in the plane which satisfies at least one of the following two conditions: (a) $f$ and $f^{\prime \prime}$ have finitely many non-real zeros and poles; (b) $f$ and $f^{(m)}$ have finitely many non-real zeros, for some $m \geq 3$. Then the Tsuji characteristic $T_{0}\left(r, f^{\prime} / f\right)$ in the upper half-plane satisfies $T_{0}\left(r, f^{\prime} / f\right)=O(\log r)$ as $r \rightarrow \infty$. 
The following lemma is due to Levin and Ostrovskii [26] (see also [2, 9] and [24, Lemma 2.2]).

Lemma 2.3 ([26]) Let $H$ be a non-constant meromorphic function in the plane. If $H$ and $G=\widetilde{H}$ satisfy, as $r \rightarrow \infty$,

$$
N(r, H)=O(r \log r) \quad \text { and } \quad T_{0}(r, H)+T_{0}(r, G)=O(\log r),
$$

then $T(r, H)=O(r \log r)$ as $r \rightarrow \infty$.

Lemma 2.4 Let $0<\varepsilon<\pi / 8, R>0$ and $K>1$. Let $\left(h_{n}\right)$ be a sequence of meromorphic functions on the domain $\{z \in \mathbb{C}:|z|>R, 0<\arg z<\pi\}$, each of them such that $h_{n}, h_{n}^{\prime}$ and $h_{n}^{\prime \prime}$ have no zeros there. Suppose that there exists a positive sequence $\left(r_{n}\right)$ such that $r_{n} \rightarrow \infty$ and

$$
\min \left\{\left|z \frac{h_{n}^{\prime}(z)}{h_{n}(z)}\right|: K^{-1} r_{n} \leq|z| \leq K r_{n}, \varepsilon \leq \arg z \leq \pi-\varepsilon\right\} \rightarrow 0
$$

as $n \rightarrow \infty$. Then

$$
\max \left\{\left|z \frac{h_{n}^{\prime}(z)}{h_{n}(z)}\right|: K^{-1} r_{n} \leq|z| \leq K r_{n}, \varepsilon \leq \arg z \leq \pi-\varepsilon\right\} \rightarrow 0
$$

as $n \rightarrow \infty$.

Proof. For $q=1,2$ let

$$
D_{q}=\left\{z \in \mathbb{C}: K^{-q}<|z|<K^{q}, \varepsilon / q<\arg z<\pi-\varepsilon / q\right\}
$$

and let $E_{1}$ be the closure of $D_{1}$. Let $n_{0} \in \mathbb{N}$ be large. By Theorem 2.2 the functions $p_{n}(z)=$ $r_{n} h_{n}^{\prime}\left(r_{n} z\right) / h_{n}\left(r_{n} z\right), n \geq n_{0}$, form a normal family of zero-free meromorphic functions on $D_{2}$. Assuming that the assertion of the lemma is false gives, after passing to a subsequence if necessary,

$$
\liminf _{n \rightarrow \infty}\left(\sup \left\{\left|p_{n}(z)\right|: z \in E_{1}\right\}\right)>0 .
$$

On the other hand (5) implies that there exist $u_{n} \in E_{1}$ with $\lim _{n \rightarrow \infty} p_{n}\left(u_{n}\right)=0$. After taking a further subsequence, if necessary, it may be assumed that, as $n \rightarrow \infty$, the points $u_{n}$ converge to some $u^{*} \in E_{1} \subseteq D_{2}$ and the functions $p_{n}$ converge locally uniformly on $D_{2}$ to some $p$ with $p\left(u^{*}\right)=0$. Thus $p$ is meromorphic on $D_{2}$ and $p \equiv 0$ by Hurwitz' theorem, which contradicts (6).

Lemma 2.5 Let $0<\varepsilon<\pi / 8$ and $K>4$, and let $q$ be a positive integer. Then there exists $C_{1}>0$ with the following property.

Let $R \geq 1$ and let the function $h$ be meromorphic on $D_{R}=\{z \in \mathbb{C}:|z|>R, \operatorname{Im} z>0\}$, and assume that $h^{(q)}(z) \not \equiv 0$ on $D_{R}$, and that $h^{\prime}$ has no zeros in $D_{R}$. Let $\left(r_{n}\right),\left(\rho_{n}\right)$ and $\left(S_{n}\right)$ be positive sequences such that $\lim _{n \rightarrow \infty} r_{n}=\infty$ and $\lim _{n \rightarrow \infty} \rho_{n}=0$. For each $n$, assume that

$$
\max \left\{\left|z \frac{h^{(q+1)}(z)}{h^{(q)}(z)}\right|: z \in \Omega_{n}\right\} \leq \rho_{n},
$$


where $\Omega_{n}=\left\{z \in \mathbb{C}: K^{-1} r_{n} \leq|z| \leq K r_{n}, \varepsilon \leq \arg z \leq \pi-\varepsilon\right\}$, and that there exists $z_{n}$ with

$$
\frac{r_{n}}{2} \leq\left|z_{n}\right| \leq 2 r_{n}, \quad 2 \varepsilon \leq \arg z_{n} \leq \pi-2 \varepsilon, \quad\left|z_{n} \frac{h^{\prime}\left(z_{n}\right)}{h\left(z_{n}\right)}\right|<S_{n} .
$$

Then, for all sufficiently large $n$, the set

$$
\left\{\theta \in[\varepsilon, \pi-\varepsilon]:\left|t_{n} \frac{h^{\prime}\left(t_{n} e^{i \theta}\right)}{h\left(t_{n} e^{i \theta}\right)}\right|<C_{1} S_{n}\right\}, \quad t_{n}=K^{-1} r_{n},
$$

has linear measure at least $\pi / 2$.

Proof. By (7) there exists $c=c(n) \in \mathbb{C}$ such that integrating from $i K r_{n}$ to $z \in \Omega_{n}$ gives

$$
\log h^{(q)}(z)=c+o(1), \quad h^{(q)}(z)=e^{c}\left(1+\frac{\delta_{q}(z)}{q !}\right), \quad \delta_{q}(z)=o(1) .
$$

It may be assumed that $e^{c}=q$ !, since $h^{\prime} / h$ and $h^{(q+1)} / h^{(q)}$ are unchanged if $h$ is replaced on $\Omega_{n}$ by $q ! e^{-c(n)} h$. Thus repeated integration gives a monic polynomial $P=P_{q, n}$, of degree $q$, with the property that, for $j=0, \ldots, q$ and for all $z$ in $\Omega_{n}$,

$$
h^{(j)}(z)=P^{(j)}(z)+\delta_{j}(z), \quad \delta_{j}(z)=o\left(|z|^{q-j}\right), \quad \delta_{j}^{\prime}(z)=\delta_{j+1}(z)=o\left(|z|^{q-j-1}\right) ;
$$

here all these estimates hold as $n \rightarrow \infty$, uniformly on $\Omega_{n}$, and the last estimate for $j=q$ follows from (7).

Let $n$ be large and denote by $c_{j}$ positive constants which are independent of $n$. Then (8) delivers a small $c_{0}$ such that the disc $\left|z-z_{n}\right|<c_{0} r_{n}$ lies in $D_{R}$, and since $h^{\prime}$ has no zeros in $D_{R}$ it follows from the minimum principle that

$$
\min \left\{\left|w \frac{h^{\prime}(w)}{h(w)}\right|:\left|w-z_{n}\right|=s\right\} \leq S_{n} \quad \text { for all } \quad s \in\left[c_{0} r_{n} / 4, c_{0} r_{n} / 2\right] .
$$

Now let $\left\{B_{j}\right\}=\left\{B_{j, n}\right\}$ denote the collection of all zeros of $P$ and $P^{\prime}$. Let $Q_{n}$ be the closed set obtained by deleting from $\Omega_{n}$ the open discs $E_{j}$ of centre $B_{j}$ and radius $c_{1} r_{n}$, where $c_{1}$ is assumed to be small. Then $z \in Q_{n}$ gives $\left|z-B_{j}\right|>c_{2}|z|$ for every $j$, and hence

$$
|P(z)|>c_{3}|z|^{q}, \quad\left|P^{\prime}(z)\right|>c_{3}|z|^{q-1}, \quad\left|\frac{P^{\prime}(z)}{P(z)}\right|+\left|\frac{P^{\prime \prime}(z)}{P^{\prime}(z)}\right|<\frac{c_{4}}{|z|} .
$$

For $z \in Q_{n}$ it follows that $\phi=h^{\prime} / h$ satisfies

$$
\begin{aligned}
\left|\frac{\phi^{\prime}(z)}{\phi(z)}\right| & =\left|\frac{P^{\prime \prime}(z)+\delta_{2}(z)}{P^{\prime}(z)+\delta_{1}(z)}-\frac{P^{\prime}(z)+\delta_{1}(z)}{P(z)+\delta_{0}(z)}\right| \\
& =\left|\frac{P^{\prime \prime}(z)}{P^{\prime}(z)(1+o(1))}-\frac{P^{\prime}(z)}{P(z)(1+o(1))}+\frac{o(1)}{|z|}\right| \leq \frac{c_{5}}{|z|} .
\end{aligned}
$$

Provided $c_{1}$ was chosen small enough, the following exist: a real number $s_{n} \in\left[c_{0} r_{n} / 4, c_{0} r_{n} / 2\right]$ such that the circle $\left|z-z_{n}\right|=s_{n}$ meets none of the discs $E_{j}$; a real number $u_{n} \in\left[r_{n} / 3,3 r_{n}\right]$ such that the circle $|z|=u_{n}$ meets $\left|z-z_{n}\right|=s_{n}$ but none of the $E_{j}$; a set $T_{n} \subseteq[\varepsilon, \pi-\varepsilon]$, of 
linear measure at least $\pi / 2$, such that for $\theta \in T_{n}$ the line segment given by $K^{-1} r_{n} \leq|z| \leq K r_{n}$, $\arg z=\theta$, lies in $Q_{n}$. Using (9), choose $w_{n}$ with

$$
\left|w_{n}-z_{n}\right|=s_{n}, \quad\left|w_{n} \frac{h^{\prime}\left(w_{n}\right)}{h\left(w_{n}\right)}\right| \leq S_{n}
$$

For $v=t_{n} e^{i \theta}=K^{-1} r_{n} e^{i \theta}$ with $\theta \in T_{n}$, there exists a path $\Gamma_{v} \subseteq Q_{n}$, joining $v$ to $w_{n}$, which consists of part of the ray $\arg z=\theta$ and arcs of the circles $|z|=u_{n}$ and $\left|z-z_{n}\right|=s_{n}$. The path $\Gamma_{v}$ has length at most $c_{6} r_{n}$, and so integrating $\phi^{\prime} / \phi$ along $\Gamma_{v}$ gives, using (10),

$$
\left|v \frac{h^{\prime}(v)}{h(v)}\right|=|v \phi(v)|<c_{7}\left|v \phi\left(w_{n}\right)\right|=c_{7}\left|v \frac{h^{\prime}\left(w_{n}\right)}{h\left(w_{n}\right)}\right|<c_{8}\left|w_{n} \frac{h^{\prime}\left(w_{n}\right)}{h\left(w_{n}\right)}\right| \leq c_{8} S_{n} .
$$

Lemma 2.6 Let $B \in \mathbb{C}$ with $|B|=1$ and $L \in \mathbb{Z} \backslash\{-1\}$, and let

$$
F(z)=\left(e^{z}-1\right)^{L}\left(e^{z}-B\right) .
$$

If all zeros of $F^{\prime \prime}$ lie on $i \mathbb{R}=\{i x: x \in \mathbb{R}\}$ then $F$ is given by one of the following:

$$
\text { (i) } \quad F(z)=e^{2 z}-1 ; \quad(\text { ii }) \quad F(z)=e^{z}-B ; \quad(\text { iii }) \quad F(z)=\frac{1}{e^{z}-1} .
$$

Proof. Note first that $L=0$ gives (ii) immediately, while if $L=1$ then $F^{\prime \prime}(z)=4 e^{2 z}-(1+B) e^{z}$, which has zeros off $i \mathbb{R}$ unless $B=-1$, in which case $F$ is given by (i). Assume henceforth that $L \neq 0, \pm 1$, so that $L B+1 \neq 0$, since $|B|=1$. Now write $X=e^{z}$ and

$$
\begin{aligned}
F(z)= & P(X)=(X-1)^{L}(X-B) \\
F^{\prime \prime}(z)= & X P^{\prime}(X)+X^{2} P^{\prime \prime}(X) \\
= & X\left(L(X-1)^{L-1}(X-B)+(X-1)^{L}\right)+ \\
& +X^{2}\left(L(L-1)(X-1)^{L-2}(X-B)+2 L(X-1)^{L-1}\right),
\end{aligned}
$$

from which it follows that

$$
\begin{aligned}
Q(X) & =X^{-1}(X-1)^{2-L} F^{\prime \prime}(z) \\
& =X^{2}(L+1)^{2}+X\left(-3 L-2-L^{2} B\right)+L B+1
\end{aligned}
$$

satisfies $Q(0)=L B+1 \neq 0$. If $Q(C)=0$ and $C \neq 1$ then $e^{z}=C$ implies $F^{\prime \prime}(z)=0$. Hence the fact that all zeros of $F^{\prime \prime}$ lie on $i \mathbb{R}$ forces each root of $Q$ to have modulus 1 , so that

$$
|L+1|^{2}=|L B+1| \leq|L|+1,
$$

which is impossible if $L \geq 2$. Now suppose that $L=-n \leq-2$. Then $(n-1)^{2} \leq n+1$ and so $n \leq 3$, giving $L=-2,-3$. Now $L=-2$ forces $|-2 B+1|=1$ and so $B=1$, which leads to (iii). Finally, if $L=-3$ then $|-3 B+1|=4$, from which it follows that $B=-1$ and

$$
Q(X)=4 X^{2}+16 X+4
$$

which does not have two roots of modulus 1 . 
Lemma 2.7 Let $A \in \mathbb{C}$. Then all solutions of $\tan z=z-A$ are real if and only if $A \in \pi \mathbb{Z}=$ $\{n \pi: n \in \mathbb{Z}\}$.

Proof. This is proved in [18] (starting from formula (5) on p.73, with $B=1$ in the notation there). However, since the method in [18] uses [14, Lemma 8], the proof of which is lengthy, it seems worth including the following self-contained argument. First, suppose that $p(z)=\tan z$ has a fixpoint $w$ in the open upper half-plane $H^{+}$. Since $p$ maps $H^{+}$into itself, but not univalently, Schwarz' lemma implies that $\left|p^{\prime}(w)\right|<1$ and that the iterates of $p$ converge to $w$ on $H^{+}$. Hence $w$ must lie on the positive imaginary axis $i \mathbb{R}^{+}$, since $p\left(i \mathbb{R}^{+}\right) \subseteq i \mathbb{R}^{+}$, which contradicts the fact that simple estimates give $\tanh y<y$ for $y>0$. Hence all fixpoints of $\tan z$ are real and periodicity implies that so are all solutions of $\tan z=z-A$ for $A \in \pi \mathbb{Z}$.

Now suppose that all solutions of $\tan z=z-A$ are real. The real meromorphic function $g(z)=z-\tan z$ has no finite asymptotic values, and so no Picard values: thus $A \in \mathbb{R}$. Suppose that $n \in \mathbb{Z}$ and $n \pi<A<(n+1) \pi$. Then $g(x)$ is decreasing on the interval $((n+1 / 2) \pi,(n+3 / 2) \pi)$ and has a fixpoint at $(n+1) \pi$, which is a zero of $g-(n+1) \pi$ of multiplicity 3. Hence there exists a level curve $\gamma$ on which $g(z)$ is real and decreasing, which starts at $(n+1) \pi$ and enters the upper half-plane. Since $g$ has no finite asymptotic values, and all critical points of $g$ are fixpoints of $g$ in $\pi \mathbb{Z}$, the curve $\gamma$ must pass through a non-real $A$-point of $g$.

\section{Intermediate steps for Theorems 1.2 and 1.3}

Throughout this section, let $M \geq 4$ be an integer and let $f$ be a strictly non-real meromorphic function in the plane such that $f, f^{\prime}, \ldots, f^{(M+3)}$ all have finitely many zeros in $\mathbb{C} \backslash \mathbb{R}$.

Lemma 3.1 If $f$ is a polynomial then $f^{\prime} / f$ is rational and $f$ has at least one non-real zero.

Proof. This follows at once from $f$ being strictly non-real.

Assume henceforth that $f$ is not a polynomial, and let $g(z)=\widetilde{f}(z)=\overline{f(\bar{z})}$. Then Lemma 2.2 shows that the Tsuji characteristics [2, 9, 29] of $f^{\prime} / f$ and $g^{\prime} / g$ satisfy

$$
T_{0}\left(r, f^{\prime} / f\right)+T_{0}\left(r, g^{\prime} / g\right)=O(\log r) \quad \text { as } \quad r \rightarrow \infty .
$$

The lemma of the logarithmic derivative for the Tsuji characteristic [26] and the formulas

$$
\psi=\frac{\phi^{\prime}}{\phi}, \quad \frac{\phi^{\prime \prime}}{\phi^{\prime}}=\frac{\psi^{\prime}}{\psi}+\psi
$$

then deliver, for all $m \geq 0$,

$$
T_{0}\left(r, f^{(m+1)} / f^{(m)}\right)+T_{0}\left(r, g^{(m+1)} / g^{(m)}\right)=O(\log r) \quad \text { as } \quad r \rightarrow \infty .
$$

For $0 \leq m \leq M+1$ write

$$
F_{m}(z)=z-\frac{f^{(m)}(z)}{f^{(m+1)}(z)}, \quad G_{m}(z)=z-\frac{g^{(m)}(z)}{g^{(m+1)}(z)}=\widetilde{F}_{m}(z) .
$$


Lemma 3.2 Let $0 \leq m \leq M+1$. Then the functions $F_{m}$ and $G_{m}$ are non-constant, and there exists a meromorphic function $K_{m}$, with finitely many zeros and poles, such that

$$
F_{m}^{\prime}=\frac{f^{(m)} f^{(m+2)}}{\left(f^{(m+1)}\right)^{2}}=K_{m}\left(\frac{g^{(m)} g^{(m+2)}}{\left(g^{(m+1)}\right)^{2}}\right)=K_{m} G_{m}^{\prime}
$$

The function $K_{m}$ satisfies $\left|K_{m}(x)\right|=1$ for all $x \in \mathbb{R}$ and there exist a rational function $R_{m}$ and a real number $a_{m}$ such that

$$
K_{m}(z)=R_{m}(z) e^{i a_{m} z}
$$

Furthermore, if $f^{(m)}, f^{(m+1)}$ and $f^{(m+2)}$ have only real zeros, then $R_{m}$ is constant.

Proof. The first assertion holds since if $F_{m}$ is constant then $F_{m}^{\prime}$ and $f^{(m+2)}$ vanish identically. Now $K_{m}$ has finitely many zeros and poles, since $f, \ldots, f^{(M+3)}$ have finitely many non-real zeros, and $\widetilde{K}_{m}=1 / K_{m}$. Finally, (14) and Lemma 2.3 imply that (17) holds.

Lemma 3.3 For $0 \leq m \leq M+1$ :

(a) every real multiple zero of $f^{(m)}$ is a 1-point of $K_{m}$;

(b) if $K_{m}$ is constant in (16), then either $F_{m}=G_{m}$ or $f^{(m)}$ has at most one real zero, counting multiplicities;

(c) every real simple zero a of $f^{(m+1)}$ either is a multiple zero of $f^{(m)}$ or satisfies $K_{m}^{\prime}(a)=0$.

Proof. To prove (a) and (b) take a real zero $x_{0}$ of $f^{(m)}$ of multiplicity $p$. Then $x_{0}$ is a zero of $g^{(m)}$ of the same multiplicity, and a common fixpoint of $F_{m}$ and $G_{m}$. If $p \geq 2$ then

$$
F_{m}^{\prime}\left(x_{0}\right)=G_{m}^{\prime}\left(x_{0}\right)=\frac{p-1}{p}, \quad K_{m}\left(x_{0}\right)=1,
$$

which proves (a). Next, if $K_{m}$ is constant but $F_{m} \neq G_{m}$ then there exists $c_{m} \in \mathbb{C}$ such that

$$
G_{m} \neq F_{m}=K_{m} G_{m}+c_{m}
$$

so that $F_{m}$ and $G_{m}$ have at most one common fixpoint, and none at all if $K_{m}=1$. In view of (a), this proves (b).

To prove (c) take a real simple zero $a$ of $f^{(m+1)}$ which is not a zero of $f^{(m)}$. Then $a$ is a simple pole of $F_{m}$, and there exists $b \in \mathbb{C} \backslash\{0\}$ such that, as $z \rightarrow a$,

$$
F_{m}(z)=\frac{b}{z-a}+O(1), \quad F_{m}^{\prime}(z)=\frac{-b}{(z-a)^{2}}+O(1), \quad G_{m}^{\prime}(z)=\frac{-\bar{b}}{(z-a)^{2}}+O(1) .
$$

This implies that $K_{m}^{\prime}(a)=0$.

The next three lemmas will treat a number of special cases.

Lemma 3.4 Assume that $0 \leq m \leq M+1$ and at least one of the following holds:

(i) $f^{(m+1)} / f^{(m)}$ is real meromorphic;

(ii) $F_{m}=G_{m}$;

(iii) $g^{(m)}=c_{m} f^{(m)}$ for some $c_{m} \in \mathbb{C}$.

Then $f$ is a rational function with at least one non-real zero. 
Proof. It is clear from (15) that (i) implies (ii) and (ii) implies (iii). Assume therefore that (iii) holds: then $\left|c_{m}\right|=1$, because $g=\widetilde{f}$ and $f^{(m)} \not \equiv 0$. Moreover, $m \geq 1$, since $f$ is strictly non-real, and $f$ and $g$ have the same poles with the same multiplicities. Hence there exists a non-constant meromorphic function $H$ with finitely many zeros and poles such that, using (12),

$$
g=H f, \quad \widetilde{H}=\frac{1}{H}, \quad \frac{g^{\prime}}{g}-\frac{f^{\prime}}{f}=h=\frac{H^{\prime}}{H}, \quad T_{0}(r, h)=O(\log r) \quad \text { as } \quad r \rightarrow \infty .
$$

Furthermore, integration gives a polynomial $P \not \equiv 0$, of degree at most $m-1 \leq M$, with

$$
g=H f=P+c_{m} f, \quad f=\frac{P}{H-c_{m}}, \quad \frac{f^{\prime}}{f}=\frac{P^{\prime}}{P}-\frac{H^{\prime}}{H-c_{m}}=\frac{P^{\prime}}{P}-\frac{h}{1-c_{m} H^{-1}} .
$$

Hence $T_{0}(r, H)=O(\log r)$ and $T(r, H)=O(r \log r)$ as $r \rightarrow \infty$, by (12), (18) and Lemma 2.3. Thus $H(z)=T_{1}(z) e^{i a_{1} z}$, where $a_{1} \in \mathbb{R}$ and $T_{1}$ is a rational function with $\left|T_{1}(x)\right|=1$ on $\mathbb{R}$.

If $H$ is transcendental then (18) and (19) show that $f$ satisfies the hypotheses of [24, Lemma 2.5], and so $f^{\prime \prime \prime}$ has infinitely many non-real zeros, contrary to assumption. Therefore $H$ is a rational function and so is $f$. Because $H$ is non-constant and $\widetilde{H}=1 / H$, the function $H$ has at least one pole and, since $f$ and $g$ have the same poles, $f$ has at least one non-real zero.

Lemma 3.5 Assume that $f$ has finite order and finitely many poles. Then either $f^{\prime} / f$ is a rational function, or $f$ satisfies (1).

Proof. The hypotheses imply that there exist meromorphic functions $H$ and $K$, each with finitely many zeros and poles, such that

$$
g=H f, \quad g^{\prime}=H K f^{\prime}, \quad \widetilde{H}=\frac{1}{H}, \quad \widetilde{K}=\frac{1}{K} .
$$

Since $f$ is strictly non-real, $H$ is non-constant. Write

$$
h=\frac{H^{\prime}}{H}, \quad k=\frac{K^{\prime}}{K}, \quad \widetilde{h}=-h, \quad \widetilde{k}=-k .
$$

Then $h$ and $k$ have finitely many poles and so are rational functions, since $f$ has finite order. Moreover, $h$ does not vanish identically, since $H$ is non-constant, and $h^{\prime} / h$ is real.

Now (20) and (21) yield

$$
g^{\prime}=h H f+H f^{\prime}=H K f^{\prime} .
$$

Here $K-1$ cannot vanish identically because $h$ does not. It follows that

$$
L=\frac{f^{\prime}}{f}=\frac{h}{K-1} .
$$

If $K$ is a rational function, then so is $f^{\prime} / f$.

Assume henceforth that $K$, which has finitely many zeros and poles, is transcendental; then $k \not \equiv 0$ in (21). Moreover, Lemma 2.3, (12), (20) and (22) imply that $K(z)=T_{1}(z) e^{i A_{1} z}$, where $A_{1} \in \mathbb{R} \backslash\{0\}$ and $T_{1}$ is rational with $\left|T_{1}(x)\right|=1$ on $\mathbb{R}$. 
If $h= \pm k$ then (22) shows that

$$
\frac{f^{\prime}}{f}= \pm \frac{K^{\prime}}{K(K-1)}, \quad f=c(1-1 / K)^{ \pm 1}, \quad c \in \mathbb{C} \backslash\{0\},
$$

and so $f$, which has finitely many poles, must satisfy (1).

Assume henceforth that $h \neq \pm k$. Combining (13), (21) and (22) leads to

$$
\frac{f^{\prime \prime}}{f^{\prime}}=L+\frac{L^{\prime}}{L}=\frac{h}{K-1}+\frac{h^{\prime}}{h}-\frac{k K}{K-1}=\frac{h-k}{K-1}+\frac{h^{\prime}}{h}-k .
$$

Observe that none of the functions $k \pm h^{\prime} / h, h \pm h^{\prime} / h$ vanishes identically, since $h^{\prime} / h$ is real but $h$ and $k$ are not. If $|z|$ is large, then (23) shows that $z$ is a zero of $f^{\prime \prime} / f^{\prime}$ if and only if $z$ is a solution of the following equations:

$$
\frac{h-k}{K-1}=k-\frac{h^{\prime}}{h} ; \quad K-1=\frac{h-k}{k-h^{\prime} / h} ; \quad K=\frac{h-h^{\prime} / h}{k-h^{\prime} / h} .
$$

Thus $f^{\prime \prime} / f^{\prime}$ has infinitely many real zeros $x$ which satisfy, by (20) and (21),

$$
\frac{k(x)-h^{\prime}(x) / h(x)}{h(x)-h^{\prime}(x) / h(x)}=\frac{1}{K(x)}=\overline{K(x)}=\frac{-h(x)-h^{\prime}(x) / h(x)}{-k(x)-h^{\prime}(x) / h(x)}=\frac{h(x)+h^{\prime}(x) / h(x)}{k(x)+h^{\prime}(x) / h(x)} .
$$

Because $k$ and $h$ are rational functions, this forces

$$
k^{2}-\left(h^{\prime} / h\right)^{2}=h^{2}-\left(h^{\prime} / h\right)^{2}, \quad h^{2}=k^{2},
$$

contradicting the assumption that $h \neq \pm k$.

Lemma 3.6 Assume that either $f^{\prime} / f$ is a rational function or $f$ satisfies (1), and that $f, f^{\prime}, f^{\prime \prime}$ and $f^{\prime \prime \prime}$ have only real zeros. Then $f$ is given by (2).

Proof. Suppose first that $f$ satisfies (1). Then $f^{\prime \prime} / f^{\prime}$ is a rational function, and so is $F_{1}$ in (15). Moreover, the function $K_{1}$ in (16) is rational and free of zeros and poles, and so is constant, but Lemma 3.4 implies that $G_{1} \neq F_{1}$. Applying Lemma 3.3 shows that $f^{\prime}$ has at most one zero, and that any zero of $f^{\prime}$ is real and simple. Now (1) gives

$$
\frac{f^{\prime}}{f-B}=\frac{T^{\prime}}{T}+i A, \quad A \neq 0 .
$$

If $T$ is non-constant then $T^{\prime} / T$ has at least two poles in $\mathbb{C}$, since $\widetilde{T}=1 / T$, and so $f^{\prime}$ has at least two zeros in $\mathbb{C}$, counting multiplicities. This is a contradiction, and so $f$ is given by (2) (iii).

Assume henceforth that $R=f^{\prime} / f$ is a rational function. Then so are $F_{0}$ and $F_{1}$ in (15), and the same argument as in the previous paragraph shows that $K_{0}$ and $K_{1}$ are constant. However, Lemmas 3.3 and 3.4 and the fact that $f$ is strictly non-real imply the following: $G_{0} \neq F_{0}$ and $G_{1} \neq F_{1}$; neither $f^{\prime} / f$ nor $f^{\prime \prime} / f^{\prime}$ is real; any zero of $f$ is real, simple and unique, and the same applies to zeros of $f^{\prime}$ and $R$. 
Suppose first that $R(\infty)=\infty$. Then $R$, since it has at most one zero, must have form $R(z)=\alpha\left(z-x_{0}\right)$ with $x_{0} \in \mathbb{R}$ and $0 \neq \alpha \in \mathbb{C}$, so that $f^{\prime \prime}(z) / f(z)=\alpha+\alpha^{2}\left(z-x_{0}\right)^{2}$. Because $f^{\prime \prime}$ has only real zeros, $\alpha$ is real and so is $f^{\prime} / f$, a contradiction.

If $R$ is a non-zero constant, then $f$ satisfies (2)(iii). Suppose next that $R$ is non-constant, with $R(\infty) \neq 0, \infty$. Then $R$ is a Möbius transformation, since it has at most one zero. Applying a change of variables $w=a_{1} z+b_{1}$ with $a_{1}, b_{1} \in \mathbb{R}$ makes it possible to assume that the unique zero of $R$ is at the origin, and that

$$
\frac{f^{\prime}(z)}{f(z)}=R(z)=\frac{a z}{z-z_{0}}=a+\frac{a z_{0}}{z-z_{0}}, \quad \frac{f^{\prime \prime}(z)}{f(z)}=\frac{a^{2} z^{2}-a z_{0}}{\left(z-z_{0}\right)^{2}}
$$

where $a, z_{0} \in \mathbb{C} \backslash\{0\}$. Here $b=a z_{0}$ is an integer and $z_{0} \notin \mathbb{R}$, since otherwise $a$ and $f^{\prime} / f$ are real. Thus $b$ must be negative and $z_{0}$ is a pole of $f$ and a double pole of $f^{\prime \prime} / f$. Next, $z_{0} / a$ must be real and positive, since $f^{\prime \prime}$ has only real zeros, and so must $-z_{0}^{2}=-b z_{0} / a$. Now write

$$
\frac{f^{\prime \prime \prime}(z)}{f(z)}=\frac{a z\left(a^{2} z^{2}-a z_{0}\right)}{\left(z-z_{0}\right)^{3}}+\frac{2 a^{2} z}{\left(z-z_{0}\right)^{2}}-\frac{2\left(a^{2} z^{2}-a z_{0}\right)}{\left(z-z_{0}\right)^{3}}=\frac{a\left(a^{2} z^{3}-3 a z_{0} z+2 z_{0}\right)}{\left(z-z_{0}\right)^{3}} .
$$

Because $z_{0}^{2}$ and $a z_{0}$ are real, so is $a^{2}$. Since $z_{0}$ is not real, $f^{\prime \prime \prime}$ must have at least one non-real zero, a contradiction.

Assume next that $R$ has a simple zero at infinity. If $R$ has no zeros in $\mathbb{C}$ then $f / f^{\prime}=1 / R$ is a linear polynomial and $f$ satisfies (2)(i). If $R$ has a zero in $\mathbb{C}$ then it has exactly one zero and two poles there, and it may be assumed that

$$
\frac{f^{\prime}(z)}{f(z)}=R(z)=\frac{a z}{\left(z-z_{1}\right)\left(z-z_{2}\right)}, \quad a, z_{1}, z_{2} \in \mathbb{C} \backslash\{0\}, \quad z_{1} \neq z_{2} .
$$

Here the residues $r_{1}=a z_{1} /\left(z_{1}-z_{2}\right)$ and $r_{2}=a z_{2} /\left(z_{2}-z_{1}\right)$ must be integers, and $r_{1} / r_{2}=-z_{1} / z_{2}$ is real. If either residue $r_{j}$ is positive, then $z_{1}$ or $z_{2}$ is real, so that both are real, and so is $a$, contradicting the fact that $R=f^{\prime} / f$ is not real. So both $r_{j}$ are real and negative, as are $z_{1} / z_{2}$ and $a$, and $f\left(z_{1}\right)=f\left(z_{2}\right)=\infty$. Now

$$
\begin{aligned}
\frac{f^{\prime \prime}(z)}{f(z)} & =\frac{a^{2} z^{2}}{\left(z-z_{1}\right)^{2}\left(z-z_{2}\right)^{2}}+\frac{a}{\left(z-z_{1}\right)\left(z-z_{2}\right)}-\frac{a z}{\left(z-z_{1}\right)^{2}\left(z-z_{2}\right)}-\frac{a z}{\left(z-z_{1}\right)\left(z-z_{2}\right)^{2}} \\
& =\frac{a^{2} z^{2}+a\left(z-z_{1}\right)\left(z-z_{2}\right)-a z\left(z-z_{2}\right)-a z\left(z-z_{1}\right)}{\left(z-z_{1}\right)^{2}\left(z-z_{2}\right)^{2}}=\frac{\left(a^{2}-a\right) z^{2}+a z_{1} z_{2}}{\left(z-z_{1}\right)^{2}\left(z-z_{2}\right)^{2}} .
\end{aligned}
$$

Since $a<0$ this forces $z_{1} z_{2}$ to be real and positive, and so $z_{1}^{2}$ and $z_{2}^{2}$ are real and negative. Next,

$$
\begin{aligned}
\frac{f^{\prime \prime \prime}(z)}{f(z)}= & \frac{a z\left(\left(a^{2}-a\right) z^{2}+a z_{1} z_{2}\right)}{\left(z-z_{1}\right)^{3}\left(z-z_{2}\right)^{3}}+\frac{\left(a^{2}-a\right) 2 z}{\left(z-z_{1}\right)^{2}\left(z-z_{2}\right)^{2}}+ \\
& -\frac{2\left(\left(a^{2}-a\right) z^{2}+a z_{1} z_{2}\right)}{\left(z-z_{1}\right)^{3}\left(z-z_{2}\right)^{2}}-\frac{2\left(\left(a^{2}-a\right) z^{2}+a z_{1} z_{2}\right)}{\left(z-z_{1}\right)^{2}\left(z-z_{2}\right)^{3}} \\
= & \frac{\left(\left(a^{2}-a\right) z^{2}+a z_{1} z_{2}\right)\left(a z-4 z+2\left(z_{1}+z_{2}\right)\right)+\left(a^{2}-a\right) 2 z\left(z-z_{1}\right)\left(z-z_{2}\right)}{\left(z-z_{1}\right)^{3}\left(z-z_{2}\right)^{3}} \\
= & \frac{a(a-1)(a-2) z^{3}+z_{1} z_{2}\left(3 a^{2}-6 a\right) z+2 a z_{1} z_{2}\left(z_{1}+z_{2}\right)}{\left(z-z_{1}\right)^{3}\left(z-z_{2}\right)^{3}} .
\end{aligned}
$$


But $a<0$, and $f^{\prime \prime \prime} / f$ has triple poles at $z_{1}$ and $z_{2}$. Hence $f^{\prime \prime \prime} / f$ has three zeros in $\mathbb{C}$, counting multiplicities, all of them real. Because $z_{1} z_{2}$ is real, $z_{1}+z_{2}$ must be real, and so 0 . But then (24) implies that $f^{\prime} / f$ is real, a contradiction.

Finally, suppose that $R$ has a zero at $\infty$ of multiplicity at least two. Then integration of $R$ around a circle $|z|=r$ with $r$ large shows that $f$ has in $\mathbb{C}$ the same number of zeros as poles, counting multiplicities, and so exactly one of each. Hence $f$ satisfies (2)(ii).

Assume for the remainder of this section that $f$ has either infinite order of growth or infinitely many poles. Then $f^{(m+1)} / f^{(m)}$ is transcendental, for each $m \geq 0$.

Lemma 3.7 The following statements all hold.

(i) If $0 \leq m \leq M+1$ and $K_{m}$ is constant then $f^{(m)}$ has finitely many zeros.

(ii) If $0 \leq m \leq M$ and $K_{m}$ and $K_{m+1}$ are both non-constant, then $\bar{N}\left(r, 1 / f^{(m+1)}\right)=O(r)$ as $r \rightarrow \infty$.

(iii) If $0 \leq m \leq M$ and $K_{m}$ and $K_{m+1}$ are both non-constant rational functions, then $f^{(m+1)}$ has finitely many zeros;

(iv) If $0 \leq m \leq M$ and $K_{m}$ and $K_{m+1}$ are both rational functions, then $f^{(m)}$ or $f^{(m+1)}$ has finitely many zeros.

Proof. Since $f$ is transcendental by assumption, Lemma 3.4 shows that $F_{m} \neq G_{m}$ for $0 \leq m \leq$ $M+1$. Thus (i) follows from Lemma 3.3(b).

Next, assume the hypotheses of (ii), and let $x_{0}$ be a real zero of $f^{(m+1)}$. By Lemma 3.3, either $x_{0}$ is a multiple zero of $f^{(m)}$ or $f^{(m+1)}$, and hence a 1-point of $K_{m}$ or $K_{m+1}$, or $x_{0}$ is a zero of $K_{m}^{\prime}$. Now (ii) and (iii) follow, by (17), and combining (i) and (iii) gives (iv).

Lemma 3.8 There exists $\alpha>0$ such that, for $1 \leq m \leq M+2$,

$$
T\left(r, f^{(m+1)} / f^{(m)}\right)+T\left(r, g^{(m+1)} / g^{(m)}\right)<\alpha r \quad \text { as } \quad r \rightarrow \infty .
$$

Proof. If $K_{0}$ or $K_{1}$ is constant, then $f$ or $f^{\prime}$ has finitely many zeros, by Lemma 3.7. If $K_{0}$ and $K_{1}$ are both non-constant then $\bar{N}\left(r, 1 / f^{\prime}\right)=O(r)$ as $r \rightarrow \infty$. This implies that

$$
\bar{N}\left(r, 1 / f^{(m)}\right)=O(r) \quad \text { as } \quad r \rightarrow \infty
$$

holds for $m=0$ or $m=1$. Since $M \geq 4$, the same argument may be applied to $K_{4}$ and $K_{5}$ to show that (26) holds for $m=4$ or $m=5$. This delivers $p \in\{0,1\}$ and $q \in\{3,4,5\}$ such that (26) holds for $m=p$ and $m=p+q$. Now Theorem 2.1 implies that there exists $d_{1}>0$ with

$$
T\left(r, f^{(p+1)} / f^{(p)}\right) \leq d_{1}\left(\bar{N}\left(r, 1 / f^{(p)}\right)+\bar{N}\left(r, 1 / f^{(p+q)}\right)\right)+O(\log r)=O(r)
$$

as $r \rightarrow \infty$ outside a set of finite measure. This gives (25) for some $m \in\{0,1\}$ and positive $\alpha$. The existence of $\alpha>0$ such that (25) holds for $1 \leq m \leq M+2$ then follows from (13).

Lemma 3.9 Call an integer $m$ exceptional if $m \geq 0$ and $f^{(m+1)}$ has finitely many zeros. Then there exists at most one exceptional $m$. 
Proof. Suppose that there exist $m$ and $m^{\prime}$ with $0 \leq m<m^{\prime}$ such that $f^{(m+1)}$ and $f^{\left(m^{\prime}+1\right)}$ have finitely many zeros. If $m^{\prime} \geq m+2$ then $f^{(m+1)}$ has finite order and finitely many poles, by Theorem 2.1, a contradiction. If $m^{\prime}=m+1$ then the same contradiction is obtained by applying Lemma 2.1 to $f^{(m)}$, using the fact that $f^{(m+1)} / f^{(m)}$ has finitely many zeros and hence finite order by (14) and Lemma 2.3 .

Lemma 3.10 Assume that $m \in\{1, \ldots, M-2\}$ is exceptional. Then

$$
\alpha^{*}=\liminf _{r \rightarrow \infty} \frac{T\left(r, f^{(m+1)} / f^{(m)}\right)}{r} \in(0,+\infty) .
$$

Proof. Assume that $m$ is as in the statement but (27) fails. In view of (25) it must therefore be the case that $\alpha^{*}=0$ in (27). Then there exists a sequence $s_{n} \rightarrow \infty$ with $T\left(s_{n}, f^{(m+1)} / f^{(m)}\right)=o\left(s_{n}\right)$ and so, by (13), (16), (25) and the lemma of the logarithmic derivative,

$$
\sum_{j=m}^{m+4} T\left(s_{n}, f^{(j+1)} / f^{(j)}\right)=o\left(s_{n}\right), \quad T\left(s_{n}, F_{m+2}^{\prime}\right)+T\left(s_{n}, F_{m+3}^{\prime}\right)=o\left(s_{n}\right) .
$$

Since $m+2 \leq M$, it follows that $a_{m+2}=a_{m+3}=0$ in (17) and hence, by Lemma 3.7, that $f^{(m+2)}$ or $f^{(m+3)}$ has finitely many zeros, which contradicts Lemma 3.9.

Lemma 3.11 There exist a real number $M_{1}>1$ and an increasing positive sequence $\left(r_{n}\right)$ with limit $\infty$ such that, for all large $n$ and all $m \in\{1, \ldots, M\}$,

$$
T\left(2 r_{n}, f^{(m+1)} / f^{(m)}\right) \leq M_{1} T\left(r_{n}, f^{(m+1)} / f^{(m)}\right) .
$$

Proof. Let $M_{2}>1$. By (25) and a growth lemma of Hayman [11, Lemma 4], each set

$$
X_{m}=\left\{r \geq 1: T\left(2 r, f^{(m+1)} / f^{(m)}\right)>M_{2} T\left(r, f^{(m+1)} / f^{(m)}\right)\right\}
$$

has upper logarithmic density at most $d_{0}=\frac{\log 2}{\log M_{2}}$. Hence it suffices to take $M_{2}$ so large that $M d_{0}<1$, and choose a sequence $r_{n} \rightarrow \infty$ in the complement of the union of the $X_{m}$.

Lemma 3.12 Let $m \in\{1, \ldots, M\}$ and let $\varepsilon>0$.

(A) If $m$ is exceptional and $\varepsilon$ is sufficiently small, then for each large $n$ there exist $L_{m, n} \in$ $\left\{f^{(m)}, g^{(m)}\right\}$ and $v_{n}$ satisfying

$$
\left|v_{n}\right|=r_{n}, \quad 2 \varepsilon \leq \arg v_{n} \leq \pi-2 \varepsilon, \quad\left|v_{n} \frac{L_{m, n}^{\prime}\left(v_{n}\right)}{L_{m, n}\left(v_{n}\right)}\right|<\exp \left(-(1 / 4) T\left(r_{n}, f^{(m+1)} / f^{(m)}\right)\right) .
$$

(B) If $a_{m} \neq 0$ in (17) then for each large $n$ there exist $L_{m, n} \in\left\{f^{(m)}, f^{(m+1)}, g^{(m)}, g^{(m+1)}\right\}$ and $v_{n}$ satisfying

$$
\left|v_{n}-i r_{n}\right|<4, \quad\left|v_{n} \frac{L_{m, n}^{\prime}\left(v_{n}\right)}{L_{m, n}\left(v_{n}\right)}\right|<e^{-\left|a_{m}\right| r_{n} / 8}
$$


Proof. To prove (A) assume that $m$ is exceptional and let $n$ be large. Since $f^{(m+1)} / f^{(m)}$ is transcendental and has finitely many zeros, combining Lemma 3.11 with a well known estimate due to Edrei and Fuchs [6, p.322] shows that, provided $\varepsilon$ is small enough, the set

$$
\left\{\theta \in[0,2 \pi]:\left|\frac{f^{(m+1)}\left(r_{n} e^{i \theta}\right)}{f^{(m)}\left(r_{n} e^{i \theta}\right)}\right|<\exp \left(-(1 / 2) T\left(r_{n}, f^{(m+1)} / f^{(m)}\right)\right)\right\}
$$

has measure at least $16 \varepsilon$. Hence there exist $L_{m, n} \in\left\{f^{(m)}, g^{(m)}\right\}$ and $v_{n}$ such that (28) holds.

To prove (B), assume that $a_{m} \neq 0$ in (17) and again let $n$ be large. By interchanging $f$ and $g$ it may be assumed that $a_{m}>0$. This implies that

$$
\left|K_{m}(z)\right|<e^{-\left|a_{m}\right| r_{n} / 2} \quad \text { for }\left|z-i r_{n}\right|<2 \text {. }
$$

It follows immediately from (16) and (30) that, for each $z$ with $\left|z-i r_{n}\right|<2$,

$$
\text { either }(a) \quad\left|G_{m}^{\prime}(z)\right|>e^{\left|a_{m}\right| r_{n} / 4} \text { or } \quad(b) \quad\left|F_{m}^{\prime}(z)\right|<e^{-\left|a_{m}\right| r_{n} / 4}
$$

Suppose first that case (a) holds in (31), for some $z$ with $\left|z-i r_{n}\right|<2$. Because $n$ is large and $G_{m}$ has finitely many non-real poles, by (15), Cauchy's estimate for derivatives implies that there exists $v_{n}$ with $\left|v_{n}-i r_{n}\right|<4$ such that

$$
\left|G_{m}\left(v_{n}\right)\right|>e^{\left|a_{m}\right| r_{n} / 6}, \quad\left|v_{n} \frac{g^{(m+1)}\left(v_{n}\right)}{g^{(m)}\left(v_{n}\right)}\right|<e^{-\left|a_{m}\right| r_{n} / 8} .
$$

This gives (29) with $L_{m, n}=g^{(m)}$.

Now suppose that case (b) holds in (31), for all $z$ with $\left|z-i r_{n}\right|<2$, in which case

$$
\left|F_{m}(z)-F_{m}\left(i r_{n}\right)\right|<2 e^{-\left|a_{m}\right| r_{n} / 4} \quad \text { for }\left|z-i r_{n}\right|<2 \text {. }
$$

Choose $v_{n}$ with $\left|v_{n}-i r_{n}\right|<2$ such that $\left|v_{n}-F\left(i r_{n}\right)\right| \geq 1$. Then (15) and (32) imply that

$$
\left|\frac{f^{(m)}\left(v_{n}\right)}{f^{(m+1)}\left(v_{n}\right)}\right|=\left|\left(v_{n}-F_{m}\left(i r_{n}\right)\right)-\left(F_{m}\left(v_{n}\right)-F_{m}\left(i r_{n}\right)\right)\right| \geq \frac{1}{2} .
$$

It now follows from (31) that

$$
\left|v_{n} \frac{f^{(m+2)}\left(v_{n}\right)}{f^{(m+1)}\left(v_{n}\right)}\right|=\left|v_{n} F_{m}^{\prime}\left(v_{n}\right) \cdot \frac{f^{(m+1)}\left(v_{n}\right)}{f^{(m)}\left(v_{n}\right)}\right|<e^{-\left|a_{m}\right| r_{n} / 8} .
$$

Thus (29) holds with $L_{m, n}=f^{(m+1)}$.

Lemma 3.13 Let $Y$ be the set of integers $m \in\{1, \ldots, M\}$ such that either $m$ is exceptional or $a_{m} \neq 0$ in (17). Then there do not exist integers $m_{1}, m_{2}, m_{3} \in Y$ satisfying

$$
m_{2} \geq m_{1}+2, \quad m_{3} \geq m_{2}+2 .
$$


Proof. Assume that $m_{1}, m_{2}, m_{3} \in Y$ satisfy (33). If any $m_{\nu}$ is exceptional and $m_{\nu} \leq M-2$ then it is unique, by Lemma 3.9: in this case let $\alpha^{*}$ be as in (27), so that $\alpha^{*}>0$. On the other hand, if no such $m_{\nu}$ exists let $\alpha^{*}=0$. In either case the set $\left\{\alpha^{*},\left|a_{m_{1}}\right|,\left|a_{m_{2}}\right|,\left|a_{m_{3}}\right|\right\}$ has a least positive member, which will be denoted by $\beta$.

Define $S_{n}$ and $K$ by

$$
S_{n}=e^{-\beta r_{n} / 8}, \quad K=4+\frac{128 \alpha}{\beta},
$$

where $\alpha$ is as in (25), and let $\varepsilon$ be small and positive.

Apply Lemma 3.12 with $m=m_{\nu}$ and $\nu=1,2,3$; this is possible since if $a_{m_{\nu}}=0$ then $m_{\nu}$ is exceptional, by the definition of $Y$. Passing to a subsequence then allows the following assumptions to be made for $\nu=1,2,3$ and all sufficiently large $n$ : first, if $m_{\nu}$ is exceptional then (28) holds for $m=m_{\nu}$, while if $m_{\nu}$ is not exceptional then $a_{m_{\nu}} \neq 0$ and (29) holds for $m=m_{\nu}$; second, $H_{\nu}=L_{m_{\nu}, n}$ is for each $n$ the same element of the set $\left\{f^{\left(m_{\nu}\right)}, f^{\left(m_{\nu}+1\right)}, g^{\left(m_{\nu}\right)}, g^{\left(m_{\nu}+1\right)}\right\}$, with $H_{\nu} \in\left\{f^{\left(m_{\nu}\right)}, g^{\left(m_{\nu}\right)}\right\}$ if $m_{\nu}$ is exceptional. It is then possible to choose $j, k \in\{1,2,3\}$ with $j<k$ such that $H_{j}$ and $H_{k}$ are both derivatives of $f$, or both derivatives of $g$. It follows from (33) that $H_{k}=H_{j}^{(q)}$ for some $q \geq 1$.

Suppose that $m_{j}$ is exceptional. Then $H_{j}$ is $f^{\left(m_{j}\right)}$ or $g^{\left(m_{j}\right)}$ and, because $1 \leq m_{j} \leq m_{k}-2 \leq$ $M-2$, the choice of $\alpha^{*}$ and $\beta$ forces $\alpha^{*}>0$ and $\alpha^{*} \geq \beta$. Therefore, in this case, (27) yields

$$
\frac{1}{4} T\left(r_{n}, H_{j}^{\prime} / H_{j}\right) \geq \frac{\alpha^{*} r_{n}}{8} \geq \frac{\beta r_{n}}{8} \quad \text { for large } n \text {. }
$$

Thus, whether or not $m_{j}$ is exceptional, (34) and (28) or (29) give $z_{n}$ such that (8) holds with $h=H_{j}$. Moreover, since $m_{k}+1 \leq M+1$ and $f, \ldots, f^{(M+3)}$ have finitely many non-real zeros, combining Lemma 2.4 with (28) or (29) for $m=m_{k}$ gives (7), for some sequence $\rho_{n} \rightarrow 0$.

Lemma 2.5 now implies that for large $n$ the set

$$
\left\{\theta \in[\varepsilon, \pi-\varepsilon]:\left|t_{n} \frac{H_{j}^{\prime}\left(t_{n} e^{i \theta}\right)}{H_{j}\left(t_{n} e^{i \theta}\right)}\right|<C_{1} S_{n}\right\}, \quad t_{n}=K^{-1} r_{n},
$$

has linear measure at least $\pi / 2$. On combination with (34) this yields, as $n \rightarrow \infty$,

$$
m\left(t_{n}, H_{j} / H_{j}^{\prime}\right) \geq \frac{1}{4} \log \frac{1}{S_{n}}-O(1)=\frac{\beta r_{n}}{32}-O(1)=\frac{\beta K t_{n}}{32}-O(1) \geq 2 \alpha t_{n},
$$

which contradicts (25).

\section{Proof of Theorem 1.2}

Assume the hypotheses of Theorem 1.2. Then the results of Section 3 hold, with $M=9$. If $f$ has finite order and finitely many poles then both conclusions of Theorem 1.2 follow from Lemmas 3.1, 3.5 and 3.6. Assume henceforth that $f$ has infinite order or infinitely many poles and let $Y$ be as in Lemma 3.13. It will be shown that there exist integers $m_{1}, m_{2}, m_{3} \in Y$ satisfying (33), contradicting Lemma 3.13 .

Suppose first that some $m \in\{1, \ldots, 8\}$ is exceptional. Then $f^{\left(m^{\prime}+1\right)}$ has infinitely many zeros for $0 \leq m^{\prime} \neq m$, by Lemma 3.9. If $m \leq 3$ then at least one of $a_{5}$ and $a_{6}$ is non-zero 
in (17), by Lemma 3.7, as is at least one of $a_{8}$ and $a_{9}$ : thus $m_{1}=m$, while $m_{2} \in\{5,6\}$ and $m_{3} \in\{8,9\}$. Similarly, if $4 \leq m \leq 6$ then at least one of $a_{1}$ and $a_{2}$ is non-zero, as is at least one of $a_{8}$ and $a_{9}$. Furthermore, if $m \geq 7$ then at least one of $a_{1}$ and $a_{2}$ is non-zero, as is at least one of $a_{4}$ and $a_{5}$.

Suppose finally that $f^{(m+1)}$ has infinitely many zeros, for $m=1, \ldots, 8$. Then Lemma 3.7 implies that at least one of $a_{2}$ and $a_{3}$ is non-zero in (17), as are at least one of $a_{5}$ and $a_{6}$ and at least one of $a_{8}$ and $a_{9}$.

\section{Proof of Theorem 1.3}

Assume the hypotheses of Theorem 1.3. Again the results of Section 3 hold, this time with $M=6$, and by Lemmas 3.1, 3.5 and 3.6 it suffices to consider the case where $f^{(m)}$ has infinite order or infinitely many poles, for each $m \geq 0$. Lemmas 3.2 and 3.7 imply that if $0 \leq m \leq 7$ and $a_{m}=0$ in (17) then $K_{m}$ in (16) is constant and $f^{(m)}$ has finitely many zeros.

The following argument gives integers $m_{1}, m_{2}, m_{3} \in Y$ satisfying (33), where $Y$ is as in Lemma 3.13, and so delivers a contradiction. Suppose first that some $m \in\{1, \ldots, 5\}$ is exceptional, so that $f^{\left(m^{\prime}+1\right)}$ has infinitely many zeros for $0 \leq m^{\prime} \neq m$, by Lemma 3.9. This implies that if $m \leq 2$ then $a_{4} a_{6} \neq 0$, by Lemma 3.7, while if $3 \leq m \leq 4$ then $a_{1} a_{6} \neq 0$, and if $m=5$ then $a_{1} a_{3} \neq 0$. On the other hand, if no $m \in\{1, \ldots, 5\}$ is exceptional, then $f^{(m+1)}$ has infinitely many zeros, for $m=1, \ldots, 5$, and $a_{2} a_{4} a_{6} \neq 0$.

\section{Proof of Theorem 1.4}

Let $f$ be a strictly non-real meromorphic function in the plane such that all but finitely many zeros and poles of $f$ and $f^{\prime \prime}$ are real. Write

$$
g=\tilde{f}, \quad \frac{f^{\prime}}{f}=\alpha+i \beta, \quad \frac{g^{\prime}}{g}=\alpha-i \beta, \quad 2 \alpha=\frac{f^{\prime}}{f}+\frac{g^{\prime}}{g}, \quad 2 i \beta=\frac{f^{\prime}}{f}-\frac{g^{\prime}}{g},
$$

in which $\alpha$ and $\beta$ are real meromorphic functions. Here $\beta$ is not identically zero, since $f / g$ is non-constant, but $\beta$ has finitely many poles. Furthermore, all poles of $\alpha$ are simple, and all but finitely many are real zeros or poles of $f$. Since $f^{\prime \prime} / f$ and $g^{\prime \prime} / g$ have, with finitely many exceptions, the same zeros and poles there exists a meromorphic function $H$ with finitely many zeros and poles such that

$$
\begin{aligned}
\frac{f^{\prime \prime}}{f} & =\alpha^{\prime}+\alpha^{2}-\beta^{2}+i\left(\beta^{\prime}+2 \alpha \beta\right)=\frac{H g^{\prime \prime}}{g} \\
& =H\left(\alpha^{\prime}+\alpha^{2}-\beta^{2}-i\left(\beta^{\prime}+2 \alpha \beta\right)\right), \quad \widetilde{H}=\frac{1}{H} .
\end{aligned}
$$

In view of Lemmas 2.2 and 2.3, as well as standard properties of the Tsuji characteristic,

$$
T_{0}\left(r, f^{\prime} / f\right)+T_{0}\left(r, g^{\prime} / g\right)+T_{0}(r, \beta)=O(\log r) \quad \text { and } \quad T(r, \beta)+T(r, H)=O(r \log r)
$$

as $r \rightarrow \infty$. If $f$ has finite lower order then $\beta$ is a rational function. 
Now $H \equiv 1$ implies that $f^{\prime \prime} / f$ is real meromorphic and $f^{\prime} / f$ is a rational function, by [24, Theorem 1.3], and so (3) evidently holds: moreover, the same result shows that if, in addition, $f$ and $f^{\prime \prime}$ have only real zeros and poles then $f$ satisfies (4)(a).

Assume henceforth that $H \not \equiv 1$. Then rearranging (36) delivers

$$
\alpha^{\prime}+\alpha^{2}-\beta^{2}=C\left(\beta^{\prime}+2 \alpha \beta\right), \quad C=i\left(\frac{H+1}{H-1}\right)
$$

in which $C$ is a real meromorphic function.

Lemma 6.1 If $z_{0} \in \mathbb{C}$ is a pole of $\alpha$ but not of $\beta$, and if $\operatorname{Res}\left(\alpha, z_{0}\right) \neq 1$, then $C\left(z_{0}\right)=\infty$. This holds in particular if $\left|z_{0}\right|$ is large and $z_{0}$ is a pole or multiple zero of $f$.

Proof. The residue condition implies that $z_{0}$ is a double pole of $\alpha^{\prime}+\alpha^{2}$, and hence a pole of $C$, by (38). The second assertion follows from (35).

Now (38) yields

$$
0=\alpha^{\prime}-C \beta^{\prime}-C^{\prime} \beta+\alpha^{2}-2 \alpha C \beta+C^{2} \beta^{2}+C^{\prime} \beta-\left(1+C^{2}\right) \beta^{2}
$$

and so

$$
0=\gamma^{\prime}+\gamma^{2}+C^{\prime} \beta-\left(1+C^{2}\right) \beta^{2}, \quad \gamma=\alpha-C \beta
$$

Lemma 6.2 Assume that $H$ is a rational function in (36). Then $f$ satisfies (3).

If, in addition, $f$ has finite lower order and all zeros and poles of $f$ and $f^{\prime \prime}$ are real, then $\beta$, $\gamma, \alpha$ and $f^{\prime} / f$ are all constants, and $f$ satisfies the first equation of (4).

Proof. Since $H$ is a rational function, so is $C$. By (35) and Lemma 6.1, all but finitely many poles of $\alpha$ are real and simple with residue 1 , and the same is true of $\gamma$ by (39). Let $x_{0}$ be large and positive, and choose $x_{1}>x_{0}$ such that $\gamma\left(x_{1}\right) \neq \infty$. The Riccati equation (39) may be linearised by writing

$$
U\left(x_{1}\right)=1, \quad \frac{U^{\prime}}{U}=\gamma, \quad U^{\prime \prime}+\left(C^{\prime} \beta-\left(1+C^{2}\right) \beta^{2}\right) U=0 .
$$

Then $U$ extends to be analytic in the half-plane $H_{0}$ given by $\operatorname{Re} z>x_{0}$, and $U$ is real on $\left(x_{0}, \infty\right)$. For $x>x_{0}$, write $C^{\prime}(x)=\rho(x) C(x)$, where $\rho(x)$ is small and real, so that

$$
1 \geq \frac{\rho^{2}}{4}-\left(C \beta-\frac{\rho}{2}\right)^{2}=\rho C \beta-C^{2} \beta^{2} \geq \rho C \beta-\left(1+C^{2}\right) \beta^{2}=C^{\prime} \beta-\left(1+C^{2}\right) \beta^{2} .
$$

Thus the Sturm comparison theorem [5, p.355] applied to $U(x)$ and $V(x)=\sin x$ implies that the number of zeros of $U$ in the interval $\left[x_{0}, x\right]$ is $O(x)$ as $x \rightarrow+\infty$, and the same is true for the number of poles of $\gamma$, and hence of $\alpha$ and $f^{\prime} / f$, by (35) and (39). Applying a similar argument on the negative real axis proves the first estimate of (3), and the second follows using (37) and Lemma 2.3 .

Suppose in addition that $f$ has finite lower order and all zeros and poles of $f$ and $f^{\prime \prime}$ are real. Then $\beta$ is a polynomial in (35), and the rational function $H$ is free of zeros and poles, and so is constant, as is $C$. Moreover, all poles of $\gamma$ are real and simple with residue 1 , so that 
$U$ is now a real entire function, with only real zeros, of finite order by (40). Furthermore, $U$ has at most one zero, by the Sturm comparison theorem applied to $U(x)$ and $V(x)=1$. Thus $\gamma=\alpha-C \beta=U^{\prime} / U$ has at most one pole, and so is a rational function. Hence there exist a polynomial $K=\beta \sqrt{1+C^{2}} \not \equiv 0$ and a constant $\eta= \pm 1$ such that, as $z \rightarrow \infty$, (39) delivers $\gamma(z)=O(|K(z)|)$ and

$$
\begin{aligned}
K(z)^{2} & =\gamma(z)^{2}+\gamma(z) \cdot \frac{O(1)}{z}=\gamma(z)^{2}+K(z) \cdot \frac{O(1)}{z}, \\
\gamma(z) & =\eta K(z)+X(z)=\eta K(z)+\frac{O(1)}{z}, \\
0 & =\eta K^{\prime}(z)+X^{\prime}(z)+2 \eta K(z) X(z)+X(z)^{2}=\eta K^{\prime}(z)+2 \eta K(z) X(z)+\frac{O(1)}{z^{2}},
\end{aligned}
$$

as well as

$$
\frac{U^{\prime}(z)}{U(z)}+\frac{K^{\prime}(z)}{2 K(z)}=\gamma(z)+\frac{K^{\prime}(z)}{2 K(z)}=\eta K(z)+X(z)+\frac{K^{\prime}(z)}{2 K(z)}=\eta K(z)+\frac{O(1)}{z^{2} K(z)} .
$$

The argument principle now shows that $U$ and $K$ have no zeros, and hence $K$ and $\beta$ are constant, while $\gamma$ is a polynomial and is also constant, as are $\alpha$ and $f^{\prime} / f$.

Assume henceforth that $H$ is transcendental in (36). The next lemma follows immediately from (37).

Lemma 6.3 There exist $a \in \mathbb{R} \backslash\{0\}$ and a rational function $T$ with $|T(x)|=1$ for all $x \in \mathbb{R}$, such that $H(z)=T(z) e^{i a z}$.

It may be assumed that $a=2$ and $T(\infty)=1$ in Lemma [6.3, so that (38) gives

$$
H(z)=e^{2 i \zeta(z)}, \quad \zeta(z)=z+\frac{\log T(z)}{2 i}, \quad C(z)=i\left(\frac{H(z)+1}{H(z)-1}\right)=\cot \zeta(z),
$$

in which the logarithm is the principal branch, while $\zeta(z)$ is analytic near infinity with $\widetilde{\zeta}=\zeta$ there. Thus (39) becomes

$$
0=\gamma^{\prime}+\gamma^{2}-\left(1+C^{2}\right)\left(\beta \zeta^{\prime}+\beta^{2}\right)=\gamma^{\prime}+\gamma^{2}-\left(\beta \zeta^{\prime}+\beta^{2}\right) S^{2}, \quad S=\frac{1}{\sin \zeta} .
$$

Lemma 6.4 Let $x_{0}$ be large and positive and let $I \subseteq \mathbb{R} \backslash\left[-x_{0}, x_{0}\right]$ be an open interval containing no poles of $S(z)$. Then $I$ contains at most one pole of $f^{\prime} / f$.

Proof. Choose $x_{1} \in I$ such that $\gamma\left(x_{1}\right) \neq \infty$ and linearise (42) near $x_{1}$ by writing

$$
u\left(x_{1}\right)=1, \quad \frac{u^{\prime}}{u}=\gamma, \quad u^{\prime \prime}+A u=0, \quad A=-\left(\beta \zeta^{\prime}+\beta^{2}\right) S^{2} .
$$

Thus $u$ extends to be analytic on a domain containing $I$, and $u$ is real-valued on $I$. Define a zero-free comparison function $v$ on $I$ by $v\left(x_{1}\right)=1$ and

$$
\frac{v^{\prime}}{v}=\frac{\zeta^{\prime} \cot \zeta}{2}-\frac{\zeta^{\prime \prime}}{2 \zeta^{\prime}}=\frac{\zeta^{\prime} C}{2}-\frac{\zeta^{\prime \prime}}{2 \zeta^{\prime}}
$$


so that

$$
\begin{aligned}
\frac{v^{\prime \prime}}{v} & =\frac{\zeta^{\prime \prime} C}{2}-\frac{\left(\zeta^{\prime}\right)^{2}\left(1+C^{2}\right)}{2}-\frac{\zeta^{\prime \prime \prime}}{2 \zeta^{\prime}}+\frac{\left(\zeta^{\prime \prime}\right)^{2}}{2\left(\zeta^{\prime}\right)^{2}}+\frac{\left(\zeta^{\prime}\right)^{2} C^{2}}{4}-\frac{\zeta^{\prime \prime} C}{2}+\frac{\left(\zeta^{\prime \prime}\right)^{2}}{4\left(\zeta^{\prime}\right)^{2}} \\
& =-\frac{\left(\zeta^{\prime}\right)^{2}\left(1+C^{2}\right)}{2}+\frac{\left(\zeta^{\prime}\right)^{2}\left(1+C^{2}-1\right)}{4}-\frac{\zeta^{\prime \prime \prime}}{2 \zeta^{\prime}}+\frac{3\left(\zeta^{\prime \prime}\right)^{2}}{4\left(\zeta^{\prime}\right)^{2}} \\
& =-\frac{\left(\zeta^{\prime}\right)^{2} S^{2}}{4}-\frac{\left(\zeta^{\prime}\right)^{2}}{4}-\frac{\zeta^{\prime \prime \prime}}{2 \zeta^{\prime}}+\frac{3\left(\zeta^{\prime \prime}\right)^{2}}{4\left(\zeta^{\prime}\right)^{2}} .
\end{aligned}
$$

Since $\zeta^{\prime}$ is a real rational function with $\zeta^{\prime}(\infty)=1$ and $x_{0}$ is large, this gives

$$
A=-\left(\beta \zeta^{\prime}+\beta^{2}\right) S^{2}=-\left(\left(\beta+\frac{\zeta^{\prime}}{2}\right)^{2}-\frac{\left(\zeta^{\prime}\right)^{2}}{4}\right) S^{2} \leq \frac{\left(\zeta^{\prime}\right)^{2} S^{2}}{4} \leq-\frac{v^{\prime \prime}}{v}
$$

on $I$. The Sturm comparison theorem [5] now implies that $u$ has at most one zero in $I$, so that $\gamma$ has at most one pole there, as have $\alpha$ and $f^{\prime} / f$, by (35) and (39).

Since poles of $S$ are poles of $C$ and zeros of $H-1$, Lemmas 6.1, 6.3 and 6.4 imply that $f$ satisfies the first estimate of (3), from which the second follows using (37) and Lemma 2.3 .

To complete the proof of Theorem [1.4, assume henceforth that $f$ has finite lower order, all zeros and poles of $f$ and $f^{\prime \prime}$ are real and $H$ is transcendental. Then $\beta$ is a polynomial, of degree $d$ say. Furthermore, $H$ is free of zeros and poles, so that it may be assumed that $H(z)=e^{2 i z}$, while $\zeta(z)=z$ and $C(z)=\cot z$. Since $\zeta^{\prime \prime} \equiv 0$, the next lemma follows from (38), (39), Lemma 6.1 and an argument identical to that in Lemma 6.4.

Lemma 6.5 (i) Any pole of $f^{\prime} / f$ in $\mathbb{C} \backslash \pi \mathbb{Z}$ is a simple zero of $f$.

(ii) If $z_{0} \in \pi \mathbb{Z}$ is a pole of $f^{\prime} / f$ then $\operatorname{Res}\left(f^{\prime} / f, z_{0}\right)=2 \beta\left(z_{0}\right)+1$.

(iii) If $n \in \mathbb{Z}$ then $f^{\prime} / f$ has in $I_{n}=(n \pi,(n+1) \pi) \subseteq \mathbb{R}$ at most one pole.

(iv) $f$ satisfies

$$
N(r, f)+N(r, 1 / f)=O\left(r^{d+1}\right) \quad \text { as } r \rightarrow \infty .
$$

Now fix $x_{1} \in I_{0}=(0, \pi)$ with $\gamma\left(x_{1}\right) \neq \infty$ and linearise (42) via $u\left(x_{1}\right)=1$ and $u^{\prime} / u=\gamma$, so that $u$ solves

$$
u^{\prime \prime}+A u=0, \quad A(z)=-\frac{\beta(z)(\beta(z)+1)}{\sin ^{2} z} .
$$

Then $u$ extends to be analytic in $\Omega=\mathbb{C} \backslash\{n \pi-i t: n \in \mathbb{Z}, t \in[0,+\infty)\}$, with $u$ real on $I_{0}$.

Lemma 6.6 Let $0<\varepsilon<\pi / 4$ and denote by $E_{0}(z)$ any term which satisfies $\log ^{+}\left|E_{0}(z)\right|=o(|z|)$ as $z \rightarrow \infty$ with $\varepsilon<\arg z<\pi-\varepsilon$. Then there exists a polynomial $P \not \equiv 0$ of degree at most 1 such that

$$
\frac{u^{\prime \prime}(z)}{u(z)}=E_{0}(z) e^{2 i z}, \quad u(z)=P(z)+E_{0}(z) e^{2 i z}, \quad \gamma(z)=\frac{P^{\prime}(z)}{P(z)}+E_{0}(z) e^{2 i z} .
$$

Proof. The first estimate follows from (44) and the remaining two are proved by the method of Gronwall's lemma, exactly as in [24, Lemma 4.3]. 
Lemma 6.7 The order of $f$ is at most $d+1$.

Proof. (43) makes it possible to write $f=\Pi e^{Q}$ where $\Pi$ is a meromorphic function with real zeros and poles and order at most $d+1$, while $Q$ must be a polynomial. It follows from (35), (39), (45) and standard estimates for logarithmic derivatives that, as $z \rightarrow \infty$ with $\varepsilon<\arg z<\pi-\varepsilon$,

$$
Q^{\prime}(z)=\frac{f^{\prime}(z)}{f(z)}-\frac{\Pi^{\prime}(z)}{\Pi(z)}=\gamma(z)+(\cot z+i) \beta(z)-\frac{\Pi^{\prime}(z)}{\Pi(z)}=O\left(|z|^{d+1 / 2}\right),
$$

so that $Q$ has degree at most $d+1$.

Lemma 6.8 If the degree $d$ of $\beta$ is positive then, as $x \rightarrow+\infty$ with $x \in \mathbb{R}$,

$$
\left|\left(\frac{f^{\prime}}{f}\right)^{\prime}(x+i)\right|+\left|\alpha^{\prime}(x+i)\right|+\left|\gamma^{\prime}(x+i)\right|=o(|(x+i) \beta(x+i)|)=o\left(|\beta(x+i)|^{2}\right) .
$$

Proof. It suffices by (35) and (39) to prove that $\left(f^{\prime} / f\right)^{\prime}(x+i)=o(|(x+i) \beta(x+i)|)$. Let $x \in(0,+\infty)$ be large, set $w=x+i$ and take $R \in[2|w|, 2|w|+1]$ such that $f(z) \neq 0, \infty$ on $|z|=R$. Denote by $a_{j}$ the zeros and poles of $f$ in $|z|<R$, repeated according to multiplicity. Applying the twice differentiated Poisson-Jensen formula [10, (1.17)] to $f$ in the disc $|z|<R$ gives

$$
\left|\left(\frac{f^{\prime}}{f}\right)^{\prime}(w)\right| \leq \frac{2}{\pi} \int_{0}^{2 \pi} \frac{R|\log | f\left(R e^{i t}\right)||}{\left|R e^{i t}-w\right|^{3}} d t+\sum\left(\frac{1}{\left|a_{j}-w\right|^{2}}+\frac{\left|a_{j}\right|^{2}}{\left|R^{2}-\overline{a_{j}} w\right|^{2}}\right),
$$

in which $\left|R e^{i t}-w\right| \geq R / 2$, while $\left|R^{2}-\overline{a_{j}} w\right| \geq(1 / 2) R^{2}$ and $\left|a_{j}-w\right| \geq 1$. Lemma 6.5 implies that the number of distinct zeros and poles of $f$ in the interval $[x-R / \log R, x+R / \log R]$ is $O(R / \log R)$, and that each of these has multiplicity at most $4 M(R, \beta)$. It now follows from Lemma 6.7 that

$$
\begin{aligned}
\left|\left(\frac{f^{\prime}}{f}\right)^{\prime}(w)\right| \leq & \frac{32}{R^{2}}(m(R, f)+m(R, 1 / f))+O\left(\frac{R M(R, \beta)}{\log R}\right)+ \\
& +(n(R, f)+n(R, 1 / f))\left(\frac{(\log R)^{2}}{R^{2}}+\frac{4}{R^{2}}\right) \\
\leq & O\left(R^{d}\right)+O\left(\frac{R M(R, \beta)}{\log R}\right)=o(R M(R, \beta))=o(|w \beta(w)|) .
\end{aligned}
$$

Lemma 6.9 The polynomial $\beta$ has degree $d=0$ and, without loss of generality, there exists a real meromorphic function $W$ on $\mathbb{C}$ of order at most 1 such that

$$
f(z)=W(z) e^{i \beta z}, \quad \frac{W^{\prime}}{W}=\alpha=\gamma+\beta C=\frac{u^{\prime}}{u}+\beta C .
$$


Proof. Assume that $\beta$ has positive degree $d$ and let $\varepsilon$ be small and positive. The equations (35) and (39) and the fact that $f$ has finite order give $M_{2}>0$ and arbitrarily large positive $R$ with $\gamma(z)=O\left(R^{M_{2}}\right)$ on $|z|=R$. Now Lemma 6.6 shows that

$$
\frac{\left(\gamma(z)-P^{\prime}(z) / P(z)\right) \sin z}{\beta(z)} \rightarrow 0
$$

as $z \rightarrow \infty$ with $\arg z=2 \varepsilon$, whereas (44) and Lemma 6.8 imply that

$$
\gamma(x+i) \sim \pm \frac{\beta(x+i)}{\sin (x+i)}, \quad \frac{\left(\gamma(x+i)-P^{\prime}(x+i) / P(x+i)\right) \sin (x+i)}{\beta(x+i)} \rightarrow \pm 1
$$

as $x \rightarrow+\infty$ with $x \in \mathbb{R}$. Since $\gamma$ has only real poles, this contradicts the Phragmén-Lindelöf principle. The remaining assertions follow from (35), (39) and Lemma 6.7.

Lemma 6.10 If $u(z)$ and $u(z+\pi)$ are linearly dependent on $\Omega$ then $f$ satisfies (4).

Proof. The hypotheses imply that $\gamma=u^{\prime} / u$ has period $\pi$ and so have the sequences of poles and zeros of $f$, by (35) and (39). Thus, by Lemma 6.5, either $f$ has in each interval $I_{n}=$ $(n \pi,(n+1) \pi), n \in \mathbb{Z}$, exactly one simple zero and no poles, or $f$ has no zeros and poles in the $I_{n}$. Moreover, the residue of $f^{\prime} / f$ at each zero of $\sin z$ is a fixed integer $m$, possibly 0 . It follows that $f$ has a representation

$$
f(z)=\left(e^{2 i z}-1\right)^{L}\left(e^{2 i z}-E\right) e^{p z+q}, \quad L \in \mathbb{Z}, \quad E, p, q \in \mathbb{C}, \quad|E|=1,
$$

in which $E=1$ is not excluded. This implies in view of (35) and (39) that, as $z \rightarrow \infty$ in $\varepsilon<\arg z<\pi-\varepsilon$,

$$
\frac{f^{\prime}(z)}{f(z)}=p+o(1), \quad \alpha(z)=p-i \beta+o(1), \quad \gamma(z)=\alpha(z)-\beta \cot z=p+o(1),
$$

so that $p=0$ by Lemma 6.6. Now $L \neq-1$ in (47), since $f$ is strictly non-real, and $f$ is determined by applying Lemma 2.6 to $F(z)=e^{-q} f(z / 2 i)$.

Assume henceforth that $u(z)$ and $u(z+\pi)$ are linearly independent solutions on $\Omega$ of (44). The proof of Theorem 1.4 will be completed by first considering certain values of $\beta$ with $|\beta|$ small, following which the remaining possibilities for $\beta$ will be disposed of together.

Lemma 6.11 If $\beta \in\{-2,-1,1\}$ then $f$ satisfies (4)).

Proof. Suppose first that $\beta=-1$ : then (44) shows that $u^{\prime \prime}=0$. By (46) and the fact that $u(z)$ and $u(z+\pi)$ are linearly independent, there exists a polynomial $T_{1}$, of degree 1 , such that

$$
\gamma=\frac{u^{\prime}}{u}=\frac{T_{1}^{\prime}}{T_{1}}, \quad f(z)=W(z) e^{-i z}=\frac{T_{1}(z)}{e^{2 i z}-1} .
$$

Now (36) and (39) lead to

$$
\begin{aligned}
\frac{f^{\prime \prime}}{f} & =\gamma^{\prime}+\left(1+C^{2}\right)+\gamma^{2}-2 C \gamma+C^{2}-1-2 i(\gamma-C) \\
& =-2 C \gamma+2 C^{2}-2 i(\gamma-C)=2(C+i)(C-\gamma) .
\end{aligned}
$$


Since $f^{\prime \prime}$ has only real zeros, all zeros of $C-\gamma$ must be real. Thus the zero of $T_{1}$ belongs to $\pi \mathbb{Z}$; if this is not the case then Lemma 2.7 gives a non-real zero $z^{*}$ of $\tan z-T_{1}(z) / T_{1}^{\prime}(z)$, with $\tan \left(z^{*}\right) \neq 0, \infty$ and so $T_{1}\left(z^{*}\right) \neq 0$, which implies that $z^{*}$ is a non-real zero of $C-\gamma$, a contradiction. It follows that $f$ is given by (4) (c).

Now suppose that $\beta \in\{-2,1\}$. Then $\beta(\beta+1)=2$ and (44) solves explicitly to give $A_{1}, B_{1} \in \mathbb{C}$ with

$$
u(z)=A_{1} \cot z+B_{1}(1-z \cot z),
$$

in which $B_{1} \neq 0$ since $u(z)$ and $u(z+\pi)$ are linearly independent. Hence there exists a polynomial $T_{1}$ of degree 1 such that, in view of (46),

$$
f(z)=\left(T_{1}^{\prime}(z)-T_{1}(z) \cot z\right)(\sin z)^{\beta} e^{i \beta z} .
$$

If $\beta=1$ this gives (4)(b), and again the zero of $T_{1}$ must belong to $\pi \mathbb{Z}$ by Lemma 2.7 .

Assume now that $\beta=-2$. Then Lemma 6.5 implies that $f$ has no multiple zeros. Suppose that $x_{0} \in \mathbb{R}$ is a simple zero of $f$, and so a simple pole with residue 1 of the real meromorphic function $\alpha$. Then there exists $D_{0} \in \mathbb{R}$ such that, as $z \rightarrow x_{0}$,

$$
\frac{f^{\prime}(z)}{f(z)}=\alpha(z)+i \beta=\frac{1}{z-x_{0}}+D_{0}-2 i+O\left(\left|z-x_{0}\right|\right), \quad \frac{f^{\prime \prime}(z)}{f(z)}=\frac{2\left(D_{0}-2 i\right)}{z-x_{0}}+O(1) .
$$

This shows that $x_{0}$ is a pole of $f^{\prime \prime} / f$, and so not a zero of $f^{\prime \prime}$. Thus every zero of $f^{\prime \prime}$ must be a real zero of $f^{\prime \prime} / f$ and so of $\alpha$, by (36). But (48) leads to

$$
\alpha=\frac{f^{\prime}}{f}-i \beta=\frac{-T_{1}^{\prime} C+T_{1}\left(1+C^{2}\right)}{T_{1}^{\prime}-T_{1} C}-2 C=\frac{-3 T_{1}^{\prime} C+T_{1}+3 T_{1} C^{2}}{T_{1}^{\prime}-T_{1} C} .
$$

Hence if $|z|$ is large and $\alpha(z)=0$ then $C \neq \infty$ and $3 C^{2}+1=o(1) C$, so that $C$ is non-real and so is $z$. Therefore $f^{\prime \prime}$ has finitely many zeros and, by the main result of [21], $f$ has finitely many poles, contradicting (48).

Lemma 6.12 Let $n \in \mathbb{Z}$. Then near $n \pi$ there exist linearly independent local solutions $u_{1}, u_{2}$ of (44) of form

$$
u_{1}(z)=(z-n \pi)^{-\beta} h_{1}(z), \quad u_{2}(z)=(z-n \pi)^{\beta+1} h_{2}(z), \quad h_{j}(z)=1+\sum_{k=1}^{\infty} a_{j, k}(z-n \pi)^{k},
$$

in which the $h_{j}$ are analytic on $|z-n \pi|<\pi$ and the coefficients $a_{j, k}$ are independent of $n$. Moreover, $2 \beta+1$ is an integer, and $\beta \neq \pm 1 / 2$ and $\beta \neq-3 / 2$. Finally, if $u_{3}, u_{4}$ are non-trivial solutions on $\Omega$ of (44), then $u_{3}^{2}, u_{4}^{2}$ and $u_{3} / u_{4}$ all extend to be meromorphic in the plane.

Proof. Choose some $n \in \mathbb{Z}$ and observe first that, near the regular singular point $n \pi$, there exists $\delta \in\{-\beta, \beta+1\}$ such that (44) has a solution of form

$$
U_{1}(z)=(z-n \pi)^{\delta} H_{1}(z), \quad H_{1}(z)=1+\sum_{k=1}^{\infty} b_{k}(z-n \pi)^{k}
$$


with $H_{1}$ analytic on $|z-n \pi|<\pi$. Since $U_{1}(z+\pi)$ solves (44), for $z$ near $(n-1) \pi$, such a solution exists for any $n$, with the same choice of $b_{k}$. To obtain a further solution near $n \pi$ write

$$
\begin{aligned}
U_{2}(z) & =U_{1}(z) \int U_{1}(z)^{-2} d z=U_{1}(z) \int(z-n \pi)^{-2 \delta}\left(1-2 b_{1}(z-n \pi)+\ldots\right) d z \\
& =U_{1}(z)\left(c_{1} \log (z-n \pi)+(z-n \pi)^{1-2 \delta}\left(d_{0}+d_{1}(z-n \pi)+\ldots\right)\right),
\end{aligned}
$$

in which the series $\sum_{k=0}^{\infty} d_{k}(z-n \pi)^{k}$ is obtained by formal integration but has positive radius of convergence. Suppose first that $c_{1} \neq 0$. Then $-2 \delta \in \mathbb{Z}$, and so $U_{1}^{-2}=\left(U_{2} / U_{1}\right)^{\prime}$ has a meromorphic extension to a neighbourhood of $n \pi$, as have $\gamma=u^{\prime} / u$ and $U_{1}^{\prime} / U_{1}$. Write the solution $u$ of (44) locally in the form $u=\alpha_{1} U_{1}+\alpha_{2} U_{2}$ near $n \pi$, with the $\alpha_{j} \in \mathbb{C}$. Then the logarithmic derivative of $\alpha_{1}+\alpha_{2} U_{2} / U_{1}$ extends meromorphically to a neighbourhood of $n \pi$, and if $\alpha_{2} \neq 0$ so does $U_{2} / U_{1}$, a contradiction. Hence $u$ must locally be a constant multiple of $U_{1}$ only, so that $u(z)$ and $u(z+\pi)$ are linearly dependent, contrary to assumption.

Thus a logarithm cannot arise in (51), which forces $\beta \neq-1 / 2$ and $-\beta \neq \beta+1$, and there exist local solutions $u_{1}, u_{2}$ as in (49), obtained via (50) and (51), with the coefficients $a_{j, k}$ independent of $n$. It follows that near $n \pi$ the meromorphic function $W$ in (46) is a linear combination of

$$
v_{1}(z)=k_{1}(z), \quad v_{2}(z)=(z-n \pi)^{2 \beta+1} k_{2}(z),
$$

where the $k_{j}$ are analytic on $|z-n \pi|<\pi$, with $k_{j}(n \pi) \neq 0$. But then, if $2 \beta+1 \notin \mathbb{Z}$, it must be the case that $W$ is a constant multiple of $v_{1}$ only, so that $u(z)$ and $u(z+\pi)$ are linearly dependent, again contrary to assumption.

Next, suppose that $\beta=1 / 2$ or $\beta=-3 / 2$. Then one of $-\beta$ and $\beta+1$ is $-1 / 2$ and by (49) there exists, near 0 , a solution of (44) of form $U_{3}(z)=z^{-1 / 2}\left(1+e_{1} z+e_{2} z^{2}+\ldots\right)$, so that

$$
\begin{aligned}
\beta(\beta+1) U_{3}(z) & =\frac{3}{4} z^{-1 / 2}\left(1+e_{1} z+e_{2} z^{2}+\ldots\right)=U_{3}^{\prime \prime}(z) \sin ^{2} z \\
& =\left(\frac{3}{4} z^{-5 / 2}-\frac{1}{4} e_{1} z^{-3 / 2}+\frac{3}{4} e_{2} z^{-1 / 2}+\ldots\right)\left(z^{2}-\frac{z^{4}}{3}+\ldots\right) \\
& =\frac{3}{4} z^{-1 / 2}-\frac{1}{4} e_{1} z^{1 / 2}+z^{3 / 2}\left(\frac{3}{4} e_{2}-\frac{1}{4}\right)+\ldots
\end{aligned}
$$

Comparing the coefficients of $z^{3 / 2}$ yields a contradiction.

To complete the proof observe that, because $2 \beta+1 \in \mathbb{Z}$, the $u_{j}$ in (49) are such that $u_{1}^{2}, u_{2}^{2}$, $u_{1} u_{2}$ and $u_{1} / u_{2}$ extend to be meromorphic on a neighbourhood of $n \pi \in \pi \mathbb{Z}$.

In view of Lemmas 6.11 and 6.12, as well as the fact that $f$ is strictly non-real, it remains only to consider the case where $2 \beta+1 \in \mathbb{Z}$ but

$$
\beta \notin\{-2,-3 / 2,-1,-1 / 2,0,1 / 2,1\}, \quad \beta+1 \notin\{-1,-1 / 2,0,1 / 2,1,3 / 2,2\} .
$$

Lemma 6.13 If $n \in \mathbb{Z}$ then $u^{2}$ has at $n \pi$ a zero or pole of multiplicity at least 3.

Furthermore, there exist infinitely many $n \in \mathbb{Z}$ such that $n \pi$ is a pole of $u^{2}$, and infinitely many $n \in \mathbb{Z}$ such that $n \pi$ is a zero of $u^{2}$. 
Proof. The equation (44) has local solutions $u_{j}$ as in (49), in which $-\beta$ and $\beta+1$ have opposite signs, and $u^{2}$ has a zero or pole at $n \pi$ of multiplicity $2|\beta| \geq 3$ or $2|\beta+1| \geq 3$, by (52).

To prove the last assertion, assume that $u^{2}$ has a pole at all but finitely many $n \pi, n \in \mathbb{Z}$, or that $u^{2}$ has a zero at all but finitely many of these points. In the first case set $V=u^{2}$, and in the second set $V=u^{-2}$. Then $V$ satisfies, as $r \rightarrow \infty$,

$$
\frac{6 r}{\pi}-O(1) \leq n(r, V), \quad \frac{6 r}{\pi}-O(\log r) \leq N(r, V)
$$

On the other hand, Lemma 6.5 shows that if $n \in \mathbb{Z}$ then in the interval $I_{n}=(n \pi,(n+1) \pi)$ the function $f^{\prime} / f$ has at most one pole, and any such pole has residue 1 . The same is true of $\alpha$ and $\gamma=u^{\prime} / u$, by (35) and (39), and so $u^{2}$ has no poles and at most two zeros in $I_{n}$. This implies that, as $r \rightarrow \infty$, by (153) and Jensen's formula,

$$
N(r, 1 / V) \leq \frac{4 r}{\pi}+O(\log r) \leq\left(\frac{2}{3}+o(1)\right) N(r, V) \leq \frac{3}{4} T(r, 1 / V) .
$$

Since $f$ has finite order, applying [11, Lemma 4] gives $C_{0}>1$ and a set $E_{1} \subseteq[1, \infty)$, of positive lower logarithmic density, such that $T(2 r, 1 / V) \leq C_{0} T(r, 1 / V)$ for all $r \in E_{1}$. Choose a positive constant $\varepsilon$, so small that

$$
88 C_{0} \varepsilon\left(1+\log ^{+} \frac{1}{4 \varepsilon}\right)<\frac{1}{16} .
$$

Then Lemma 6.6, the fact that $u^{2}$ is real meromorphic and an inequality of Edrei and Fuchs [6, p.322] together deliver, for large $r \in E_{1}$,

$$
\begin{aligned}
m(r, 1 / V) & \leq O(\log r)+11\left(\frac{2 r}{2 r-r}\right) 4 \varepsilon\left(1+\log ^{+} \frac{1}{4 \varepsilon}\right) T(2 r, 1 / V) \\
& \leq O(\log r)+88 C_{0} \varepsilon\left(1+\log ^{+} \frac{1}{4 \varepsilon}\right) T(r, 1 / V) \leq \frac{1}{8} T(r, 1 / V),
\end{aligned}
$$

which contradicts (54).

Lemma 6.14 The function

$$
G(z)=\frac{u(z+\pi)-u(z)}{\pi}
$$

is a non-trivial solution of (44) with period $\pi$ on $\Omega$.

Proof. Lemma 6.6 shows that $u(z)$ is asymptotic to a polynomial $P \not \equiv 0$ of degree at most 1 as $z \rightarrow \infty$ in $\varepsilon<\arg z<\pi-\varepsilon$. The Wronskian $W_{u}$ of $u(z)$ and $u(z+\pi)$ is constant, by Abel's identity and (44). If $P$ is constant then $W_{u}$ tends to 0 in a sector and so must vanish identically, forcing $u(z)$ and $u(z+\pi)$ to be linearly dependent, contrary to assumption.

Thus $P$ must be non-constant, and $G(z)$ and $G(z+\pi)$ both solve (44) and are asymptotic to the same non-zero constant as $z \rightarrow \infty$ in $\varepsilon<\arg z<\pi-\varepsilon$. The argument of the previous paragraph now shows that $G(z)$ and $G(z+\pi)$ are linearly dependent and must be equal. 
It is now possible to write

$$
u(z)=z G(z)+K(z), \quad \frac{K(z)}{G(z)}=\frac{u(z)}{G(z)}-z,
$$

where $K$ also has period $\pi$ on $\Omega$. Moreover, $G^{2}$ and $K / G$ are meromorphic in the plane, by Lemma 6.12, and have period $\pi$. Lemma 6.13 implies that $G^{2}$ has at least one pole in $\pi \mathbb{Z}$, and so a pole at every point of $\pi \mathbb{Z}$. If $n \in \mathbb{Z}$ and $n \pi$ is not a pole of $u^{2}$ then, as $z \rightarrow n \pi$ with $z \in \Omega$,

$$
u(z)=z G(z)+K(z)=O(1), \quad \frac{K(z)}{G(z)}=\frac{u(z)}{G(z)}-z \rightarrow-n \pi,
$$

which cannot hold for more than one such $n \pi$, since $K / G$ is periodic. Thus $u^{2}$ has a pole at all but at most one $n \pi \in \pi \mathbb{Z}$, contradicting Lemma 6.13.

\section{References}

[1] W. Bergweiler and A. Eremenko, Proof of a conjecture of Pólya on the zeros of successive derivatives of real entire functions, Acta Math. 197 (2006), 145-166.

[2] W. Bergweiler, A. Eremenko and J.K. Langley, Real entire functions of infinite order and a conjecture of Wiman, Geometric and Functional Analysis 13 (2003), 975-991.

[3] W. Bergweiler and J.K. Langley, Nonvanishing derivatives and normal families, J. Analyse Math. 91 (2003), 353-367.

[4] T. Craven, G. Csordas, and W. Smith, The zeros of derivatives of entire functions and the Pólya-Wiman conjecture, Annals of Math. (2) 125 (1987), 405-431.

[5] P. Duren, Invitation to Classical Analysis, Pure and Applied Undergraduate Texts 17, Amer. Math. Soc., Providence 2012.

[6] A. Edrei and W.H.J. Fuchs, Bounds for the number of deficient values of certain classes of meromorphic functions, Proc. London Math. Soc. (3) 12 (1962), 315-344.

[7] G. Frank, W. Hennekemper and G. Polloczek, Über die Nullstellen meromorpher Funktionen und deren Ableitungen, Math. Ann. 225 (1977), 145-154.

[8] G. Frank and J.K. Langley, Pairs of linear differential polynomials, Analysis 19 (1999), 173-194.

[9] A.A. Gol'dberg and I. V. Ostrovskii, Distribution of values of meromorphic functions, Nauka, Moscow, 1970 (Russian). English transl., Transl. Math. Monogr. 236, Amer. Math. Soc., Providence 2008.

[10] W.K. Hayman, Meromorphic functions, Clarendon Press, Oxford, 1964.

[11] W.K. Hayman, On the characteristic of functions meromorphic in the plane and of their integrals, Proc. London Math. Soc. (3) 14A (1965), 93-128.

[12] S. Hellerstein, L.-C. Shen and J. Williamson, Reality of the zeros of an entire function and its derivatives, Trans. Amer. Math. Soc. 275 (1983), 319-331.

[13] S. Hellerstein, L.-C. Shen and J. Williamson, Real zeros of derivatives of meromorphic functions and solutions of second order differential equations, Trans. Amer. Math. Soc. 285 (1984), 759-776.

[14] S. Hellerstein and J. Williamson, Derivatives of entire functions and a question of Pólya, Trans. Amer. Math. Soc. 227 (1977) 227-249.

[15] A. Hinkkanen, Reality of zeros of derivatives of meromorphic functions, Ann. Acad. Sci. Fenn. 22 (1997), $1-38$. 
[16] A. Hinkkanen, Zeros of derivatives of strictly non-real meromorphic functions, Ann. Acad. Sci. Fenn. 22 (1997), 39-74.

[17] A. Hinkkanen, Iteration, level sets, and zeros of derivatives of meromorphic functions, Ann. Acad. Sci. Fenn. 23 (1998), 317-388.

[18] A. Hinkkanen and J. F. Rossi, On a problem of Hellerstein, Shen and Williamson, Proc. Amer. Math. Soc. 92 (1984), $72-74$.

[19] H. Ki and Y.-O. Kim, On the number of nonreal zeros of real entire functions and the Fourier-Pólya conjecture, Duke Math. J. 104 (2000), 45-73.

[20] J.K. Langley, Proof of a conjecture of Hayman concerning $f$ and $f^{\prime \prime}$, J. London Math. Soc. (2) 48 (1993), 500-514.

[21] J.K. Langley, The second derivative of a meromorphic function of finite order, Bulletin London Math. Soc. 35 (2003), 97-108.

[22] J.K. Langley, Non-real zeros of derivatives of real meromorphic functions, Proc. Amer. Math. Soc. 137 (2009), 3355-3367.

[23] J.K. Langley, The reciprocal of a real entire function and non-real zeros of higher derivatives, Ann. Acad. Sci. Fenn. 38 (2013), 855-871.

[24] J.K. Langley, Non-real zeros of derivatives of meromorphic functions, J. Analyse Math. 133 (2017), 183-228.

[25] J.K. Langley, Transcendental singularities for a meromorphic function with logarithmic derivative of finite lower order, Comput. Methods Funct. Theory 19 (2019), 117-133.

[26] B.Ja. Levin and I.V. Ostrovskii, The dependence of the growth of an entire function on the distribution of zeros of its derivatives. Sibirsk. Mat. Zh. 1 (1960) 427-455. English transl., AMS Transl. (2) 32 (1963) 323-357.

[27] J. Rossi, The reciprocal of an entire function of infinite order and the distribution of the zeros of its second derivative, Trans. Amer. Math. Soc. 270 (1982), 667-683.

[28] T. Sheil-Small, On the zeros of the derivatives of real entire functions and Wiman's conjecture, Annals of Math. 129 (1989) 179-193.

[29] M. Tsuji, On Borel's directions of meromorphic functions of finite order, I, Tôhoku Math. J. 2 (1950) 97-112.

School of Mathematical Sciences, University of Nottingham, NG7 2RD, UK.

james.langley@nottingham.ac.uk 